\title{
Regional Hydro-geophysical Study of the Groundwater potentials of the Imo River Basin Southeastern Nigeria using Surficial Resistivity Data
}

\author{
Terhemba Theophilus Emberga., ${ }^{1}$ Alexander Iheanyichukwu Opara ${ }^{2}$, , Samuel Okechukwu Onyekuru ${ }^{2}$, Ibe \\ Alexander Omenikolo ${ }^{1}$., Onwe Rock Nkpuma ${ }^{3}$ and Eluwa, Ndidiamaka Nchedo $^{3}$
}

${ }^{1}$ Department of Physics/Electronics, Federal Polytechnic Nekede, Owerri, Nigeria.

${ }^{2}$ Department of Geology, Federal University of Technology, PMB 1526 Owerri, Nigeria.

${ }^{3}$ Department of Geology/Geophysics, Federal University Ndufu Alike Ikwo, Abakiliki, Nigeria

Correspondence Author: Terhemba Theophilus Emberga, Department of Physics/Electronics, Federal Polytechnic Nekede, Owerri, Nigeria.

E-mail:- terhemba4sure@yahoo.com

Received date: 15 June 2019, Accepted date: 23 August 2019, Online date: 29 August 2019

Copyright: ( 2019Terhemba Theophilus Emberga et al, This is an open-access article distributed under the terms of the Creative Commons Attribution License, which permits unrestricted use, distribution, and reproduction in any medium, provided the original author and source are credited.

\begin{abstract}
The Imo River Basin lies between Latitudes $4^{\circ} 38^{\prime} \mathrm{N}$ and $6^{\circ} 01^{\prime} \mathrm{N}$ and between Longitudes $6^{\circ} 53^{\prime} \mathrm{E}$ and $7^{\circ} 32^{\prime} \mathrm{E}$ and covers an area of about $9100 \mathrm{~km}^{2}$.The litho-stratigraphic units within the study area include the Ajali, Nsukka, Imo Shale, Ameki, Ogwasi and Benin Formations. Regional hydro-geophysical evaluation of groundwater potentials in Imo River Basin has been carried out with the objective of delineating the aquifer units in the area of study. A total of five hundred and sixty-nine (569) Vertical Electrical soundings (VES) were carried out using the ABEM ${ }^{\mathrm{TM}}$ Terrameter (SAS) 4000.The VES data were acquired using the Schlumberger electrode configuration with a maximum current electrode spacing of $1000 \mathrm{~m}$. Out of the 569 VES data, twenty (20) parametric soundings were done at the vicinity of existing boreholes for correlative and quality control purposes. The acquired VES data were processed using the 1-D WINRESIST ${ }^{\mathrm{TM}}$ computer iterative software. Geo-electric curve types interpreted from the study area revealed a predominance of the AKH-type with about 3-10 geo-electric layers identified from the various formations. The mean values of aquifer resistivity and conductivity are $1963.2 \Omega \mathrm{m}$ and $0.00186 \mathrm{sm}$. ${ }^{1}$ respectively with the aquifer materials mainly made up silt, sand and coarse sandstones. Similarly, the mean depth to the aquifers across the study area is $\mathbf{1 1 5 . 5} \mathrm{m}$ while the mean aquifer thickness is $\mathbf{3 9 . 8} \mathrm{m}$. The findings of this result revealed that the shale/clay across the entire area increased with depth. In addition, the aquifer potentials of the study area were revealed to be variable with the aquifer type, nature and characteristics generally controlled by the underlying geology. It was therefore concluded that the southern part of the study area has a high aquifer potential when compared to the northern part. In conclusion, the Benin Formation was delineated as the formation with the highest aquifer potentials in the study area. It is therefore recommended that a detailed groundwater exploration should be carried out before siting a borehole in the study area.
\end{abstract}

Keywords: Hydro-geophysical, Aquifer resistivity, Longitudinal Conductance, Transverse Resistance, Imo River Basin

\section{INTRODUCTION}

Availability of clean and good drinking water is one of the significant challenges before most developing countries of the world today in general and Nigeria in particular. Despite the achievements of the Millennium Development Goals (MDG) in Nigeria, most parts of the country are still without potable water (Emberga., 2019). The near-total collapse of rural water schemes in the area of study has made the majority of the populace to depend on other sources of water (rainwater, surface water, etc). However, groundwater is a major source of clean drinking water worldwide and it accounts for about 98\% of the world's freshwater (Okoro et al. 2000).With the development of exploration and drilling technology, groundwater has become the choice of both domestic and industrial water supply in the world. Therefore, individuals who could afford the drilling of water boreholes have gone ahead to drill water wells for the purpose of harnessing groundwater. In most of the cases, these privately drilled boreholes were done without any form of geological/geophysical appraisal of the quantity and quality of the groundwater. In view of the afore-mentioned therefore; there is generally poor knowledge of the aquifer geometrical, hydraulic vulnerability 
Citation: Terhemba Theophilus Emberga, et al., Regional Hydro-geophysical Study of the Groundwater potentials of the Imo River Basin Southeastern Nigeria using Surficial Resistivity Data. Australian Journal of Basic and Applied Sciences, 13(8): 76-94.DOI: 10.22587/ajbas.2019.13.8.12

characteristics of the study area. This has cumulatively led to a large number of abortive and unproductive wells in the study area (Ugada et al., 2014;Ejiogu et al.,2019).

The sedimentary sequences of Southeastern Nigeria including those of the Imo River basin are known to have several aquifer units (Uma,1989). The groundwater recharge in the area of study is good due to the high average annual rainfall of about $2275 \mathrm{~mm}$ (Uma., 1989; Ejiogu et al., 2019). However, many boreholes in the area are not produced as a result of inadequate knowledge of the regional hydrogeology of the study area, lack of technical support (with respect to geophysical and geological exploration) and poor planning/execution of the drilling projects. This has led to a significant challenge in the area especially with respect to the near-complete dearth of hydrogeological data including information on the geometry and nature of the hydraulic boundaries of the aquifers been exploited(Uma., 1989;Eke et al.,2015;Ejiogu et al., 2019). Therefore, many boreholes have been drilled in the area without systematic and comprehensive studies to establish the types, nature, and lateral extent of the aquifers within the basin (Uma.,1989;Opara et al.,2012;Eke et al.,2015).

Direct current electrical resistivity method especially using the Vertical Electrical Sounding technique is one of the geophysical methods used in groundwater exploration. The subsurface information inferred from this technique give a better knowledge of the aquifer systems and a more realistic picture of the groundwater potential of any area (Okoro et al., 2006). The technique has been successfully used in investigating groundwater potentials in different geological settings around the study area (Eke et al., 2015; Ekwe and Opara.,2012). Similarly, the application of this technique to explore for groundwater in various sedimentary basins in Nigeria is well documented (Okoro, 2000). In the last few decades, there has been an increase in the application hydro-geophysical techniques in the location of potential water-bearing formations in many parts of the study area (Ejiogu et al.,2019). This study is an integrated geophysical and hydrogeological resource mapping of the study with the aim of establishing a hydro-geological database for Imo River Basin, Southeastern Nigeria. The main objectives, therefore, are to delineate the aquiferous zones in the area in addition to the estimation of the aquifer geometrical properties including the depth, thickness and lateral extent/continuity.

\subsection{Location, Physiography and Geology of the Study Area}

The Imo River Basin lies between Latitudes $4^{\circ} 38^{\prime} \mathrm{N}-6^{\circ} 01^{\prime} \mathrm{N}$ and between Longitudes $6^{\circ} 53{ }^{\prime} \mathrm{E}-7^{\circ} 32^{\prime} \mathrm{E}$ and covers an area of about $9100 \mathrm{~km}^{2}$ as shown in the Location/Topographic map of the study area (Figure 1). There are two main sub-basins within the basin: The Oramirukwa - Otamiri sub-basin and the Aba River sub-basin (Uma, 1989). The Imo River Basin is based on bedrock of a sequence of sedimentary rocks of about 5.5km thick and with ages ranging from Upper Cretaceous to Recent (Uma, 1989). The deposition of these sedimentary rocks is related to the opening of the South Atlantic Ocean and the formation of the rift-like Benue Trough of Nigeria in the Mesozoic (Emberga.,2019). Generally, there are two major classes of structures underlying the

Imo River Basin (Uma, 1989). About 80\% of the basin consists of Coastal Plain Sand, which is composed of non-indurated sediments represented by the Benin and Ogwashi-Asaba Formations, and alluvial deposits at the estuary at the Southern end of the

Imo River Basin (Uma, 1989). The remaining $20 \%$ is underlain by a series of sedimentary rock units that get younger southwestward, a direction that is parallel to the regional dip of the formations(Uma, 1989). The geology of the study area is very complex and consists of six lithostratigraphic units which consist of the Ajali, Nsukka, Imo Shale, Ameki, Ogwasi and Benin

Formations (Figure 2). The stratigraphic sequence of the Imo River Basin is shown in table1.The Benin Formation otherwise known as the coastal plain sands unconsolidated sands/sandstones with high porosity and permeability (Ekwe and Opara.,2012).

Table 1: Regional stratigraphic sequence of the Imo River Basin (Uma, 1989)

\begin{tabular}{|c|c|c|c|}
\hline Age & Formation & $\begin{array}{c}\text { Maximum Appropriate } \\
\text { Thickness }\end{array}$ & Characteristics \\
\hline Miocene-Recent & Benin & 2000 & $\begin{array}{c}\text { Unconsolidated, yellow and white sands, occasionally pebbly with lenses } \\
\text { of grey sandy clay. }\end{array}$ \\
\hline $\begin{array}{l}\text { Oligocene - } \\
\text { Miocene }\end{array}$ & $\begin{array}{l}\text { Ogwashi/ } \\
\text { Asaba }\end{array}$ & 500 & $\begin{array}{c}\text { Unconsolidated sandstones with carbonaceous mudstones, sandy clays } \\
\text { and lignite seams }\end{array}$ \\
\hline Eocene & Ameki & 1460 & $\begin{array}{l}\text { Sandstones grey to green argillaceous sandstones, shales and thin } \\
\text { limestone }\end{array}$ \\
\hline Paleocene & Imo & 1200 & $\begin{array}{l}\text { Blue to dark grey shales and subordinate } \\
\text { Sandstones. It includes two sandstone members: the Umuna and Ebenebe } \\
\text { sandstones. }\end{array}$ \\
\hline \multirow[t]{2}{*}{$\begin{array}{c}\text { Upper } \\
\text { Maestrichtian }\end{array}$} & $\begin{array}{l}\text { Nsukka } \\
\text { Formation }\end{array}$ & 350 & $\begin{array}{c}\text { White to grey coarse-to-medium-grained sandstone; carbonaceous shales; } \\
\text { sandy shales; subordinate coals; and thin limestones. }\end{array}$ \\
\hline & $\begin{array}{c}\text { Ajali } \\
\text { Sandstone }\end{array}$ & $350+$ & $\begin{array}{l}\text { Medium-to-coarse-grained sandstones, poorly consolidated with } \\
\text { subordinate white and pale grey shale bands. }\end{array}$ \\
\hline
\end{tabular}




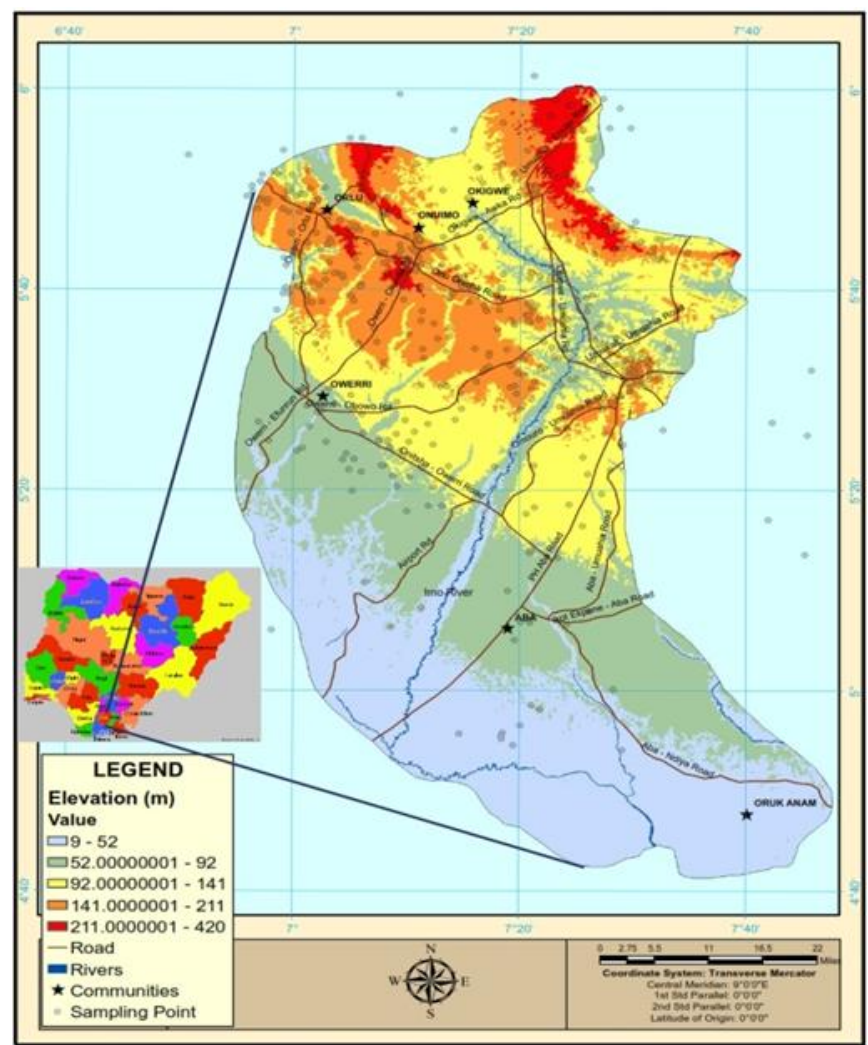

Figure 1: Location/Topographic map of the study area

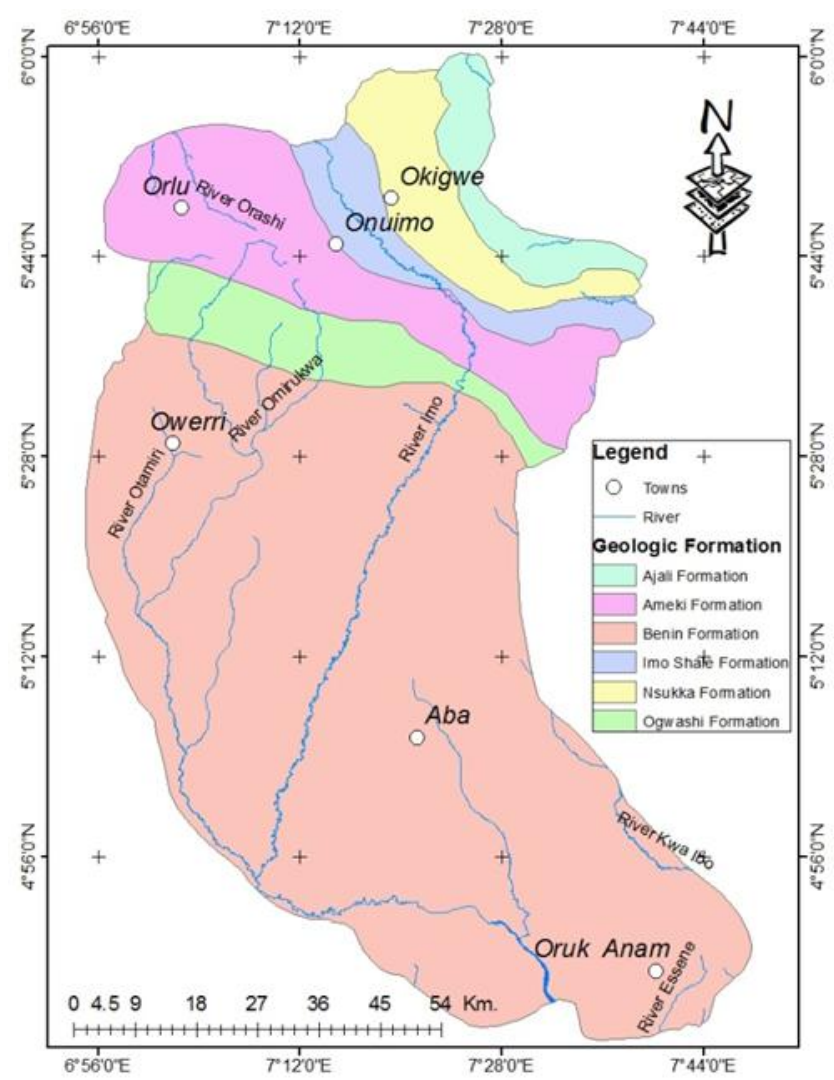

Figure 2: Geological map of Imo River Basin (Modified after Uma, 1989)

\subsection{MATERIALS AND METHODS}

A total of 569 Vertical Electrical Sounding (VES) data were acquired using the Schlumberger array with maximum current electrode separation of $500 \mathrm{~m}$. The Four (4) electrode array technique was deployed at the surface, one pair for injecting current into the ground while the other pair of electrodes are used for potential measurement. The data obtained is generally plotted as a graph of apparent resistivity against half current electrode spacing on a log-log paper. The electrode spacing at which inflexion 
Citation: Terhemba Theophilus Emberga, et al., Regional Hydro-geophysical Study of the Groundwater potentials of the Imo River Basin Southeastern Nigeria using Surficial Resistivity Data. Australian Journal of Basic and Applied Sciences, 13(8): 76-94.DOI: 10.22587/ajbas.2019.13.8.12

occurs on the graph provides an idea of the depth to the interface. A useful approximation is that the depth of the interface is equal to two-thirds (2/3) of the electrode spacing at which the point of inflexion occurs (Koefoed., 1977). This approximation has found useful applications in computer iterative modelling. The apparent resistivity was plotted on the ordinate against the half current electrode spacing to obtain the sounding curve, on a bi-logarithmic paper. Geo-electric layer parameters (i.e., apparent resistivity and thickness values) are usually obtained from the method of asymptotes as input data for computer iterative modelling (Osemeikhian et al, 1982; Igbokwe et al., 2006). Hence, the computer program makes use of the reading obtained from the field which is then converted to apparent resistivity values using computer iterative modelling.

Five hundred and sixty-nine (569) vertical electrical soundings (VES) were carried out within the study area. The data was acquired using the ABEM ${ }^{\mathrm{TM}}$ Terrameter SAS 4000 with a maximum current electrode separation of $1000 \mathrm{~m}$. For correlative and parametric purposes, twenty (20) parametric soundings were carried out at the sites of existing monitoring wells. This was done in addition to the correlation of the resistivity data with the litho-log, electric log and available pump test data from the monitoring wells across the study area. A total of five hundred and sixty-nine (569) Vertical Electrical soundings (VES) were carried out using the $\mathrm{ABEM}^{\mathrm{TM}}$ Terrameter (SAS) 4000. The VES data were acquired using the Schlumberger electrode configuration with maximum current electrode spacing of $1000 \mathrm{~m}$. Out of the 569 VES data, twenty (20) parametric soundings were carried out at the vicinity of existing boreholes for correlative and quality control purposes. The acquired VES data were later processed using the 1-D WINRESIST ${ }^{\mathrm{TM}}$ computer iterative modelling software to determine the layer parameters.

\subsection{Transverse Resistance and Longitudinal Conductance}

The transverse resistance and longitudinal conductance are two major important direct current electrical parameters that have found a considerable application in hydro-geophysical and environmental studies especially in estimating aquifer hydraulic parameters and estimation of aquifer protective capacity from surficial resistivity data. Consider a column of earth material consisting of horizontal beds, each with its own characteristic resistivity $\rho_{i}$ and thickness $h_{i}$ as shown in figure 3 below:

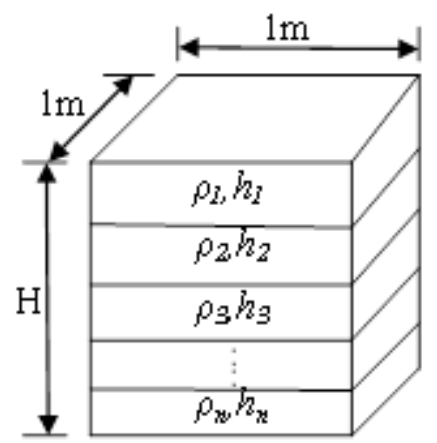

Figure 3: A 3-D Column of Rock Consisting of Horizontal Layers (Ward, 1990)

The total thickness $H=\sum_{i}^{n} h_{i} ; \quad i=1,2,3, \ldots \ldots \ldots \ldots, n$

The total resistance due to the current flowing vertically through the column is usually derived by simply adding the resistances contributed by individual layer in the column. The resistance of $i^{\text {th }}$ layer is given by

$R_{i}=\frac{\rho_{i h_{i}}}{A_{i}}=\rho_{i} h_{i} ; A_{i}=1 m^{2}$

Therefore, sum of the resistances of the individual layers known as the transverse resistance (T) is given as:

$$
T=\sum \rho_{i} h_{i} ;=1,2,3, \ldots \ldots n
$$

Combining equations $1-3$, the average transverse resistivity $\left(\rho_{t}\right)$ which is the transverse resistance per unit thickness is given by the relationship:

$$
\rho_{t}=\frac{T}{H}=\frac{\sum \rho_{i} h_{i}}{\sum h_{i}} ; i=1,2,3 \ldots \ldots n
$$

The total conductance due to the current flowing horizontally through the column is obtained by adding the conductances contributed by the individual layers in the column. However, the conductance of the $i^{\text {th }}$ layer is as:

$S_{i}=\frac{1}{R_{i}}=\frac{h_{i}}{\rho_{i}}$

Therefore, the sum of the conductance known as the longitudinal conductance (S) is given by

$$
S=\sum \frac{h_{i}}{\rho_{i}} ; i=1,2,3, \ldots \ldots n
$$

The quantities transverse resistance and longitudinal conductance $(\mathrm{S})$ are generally referred to as Dar-Zarrock parameters.

\section{RESULT AND INTERPRETATION}

\subsection{Interpretation of the Layer Parameters}

The acquired field resistivity data were used to generate geo-electric curves representative of the underlying geology of the study area. The configuration of the curves for each sounding location usually gives an understanding of the physical characteristics of the layers within such site. Several geoelectric sounding curve types were therefore interpreted from the study 
Citation: Terhemba Theophilus Emberga, et al., Regional Hydro-geophysical Study of the Groundwater potentials of the Imo River Basin Southeastern Nigeria using Surficial Resistivity Data. Australian Journal of Basic and Applied Sciences, 13(8): 76-94.DOI: 10.22587/ajbas.2019.13.8.12

area reflecting the complex geology of the study area. This is because the configuration of a VES curve is a function of the number of layers in the subsurface, the thickness of each layer, and the ratio of the resistivity of the layers (Inyang et al., 2017). Typical geo-electric curves interpreted from the study area are shown in figure 4. Curve types interpreted from the study area include the A, AH, AHA, AK, AKH, HKA, KHA etc with the dominant curve type being the AKH type as summarized in Table 2 and figure 5 .

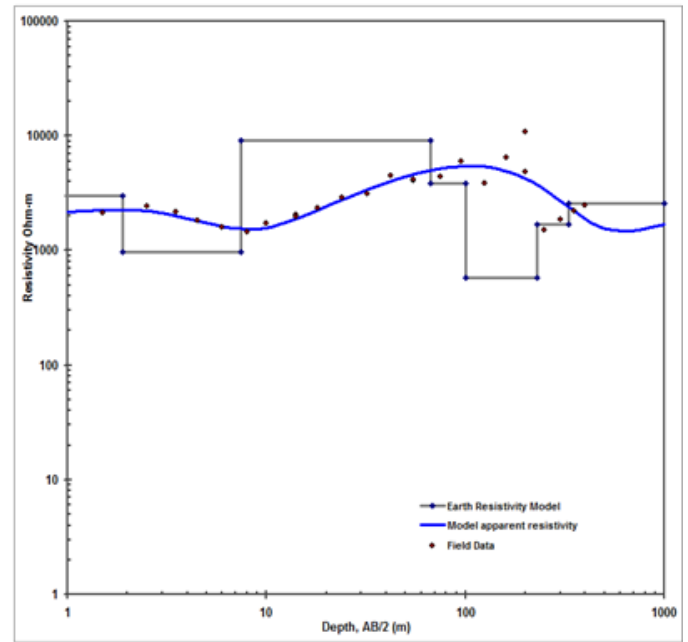

(a)

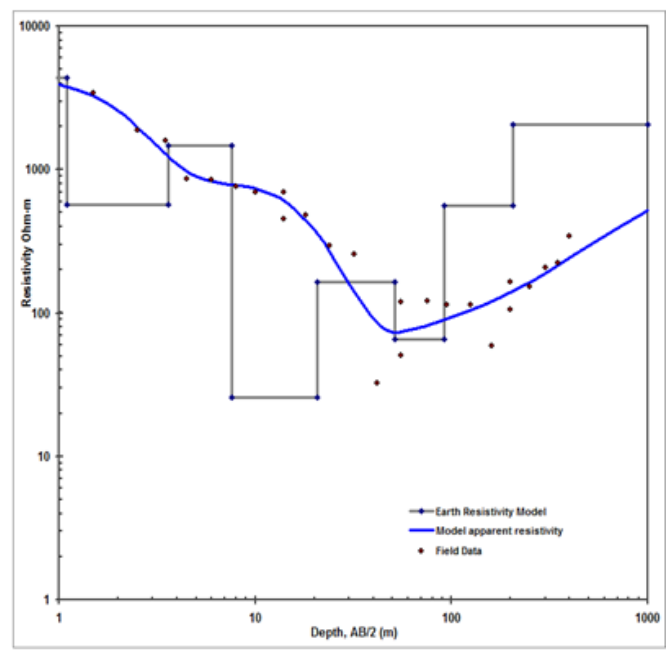

(c)

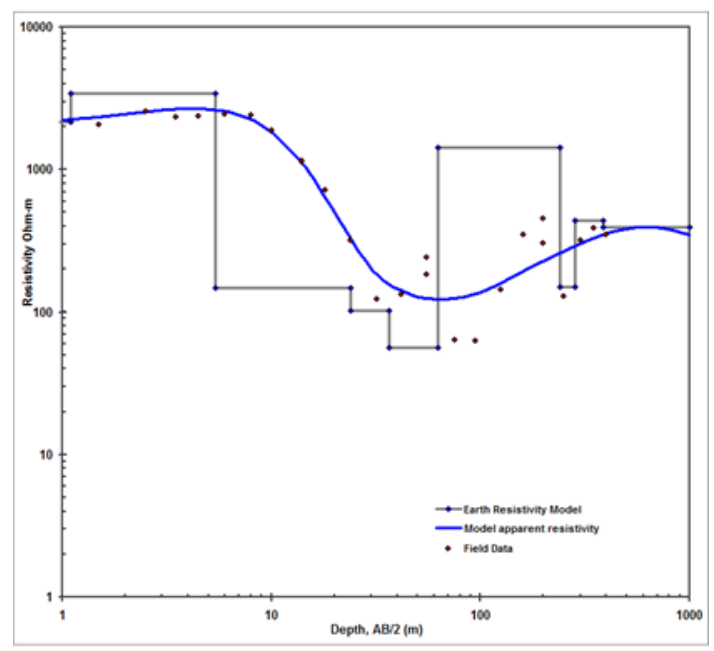

(b)

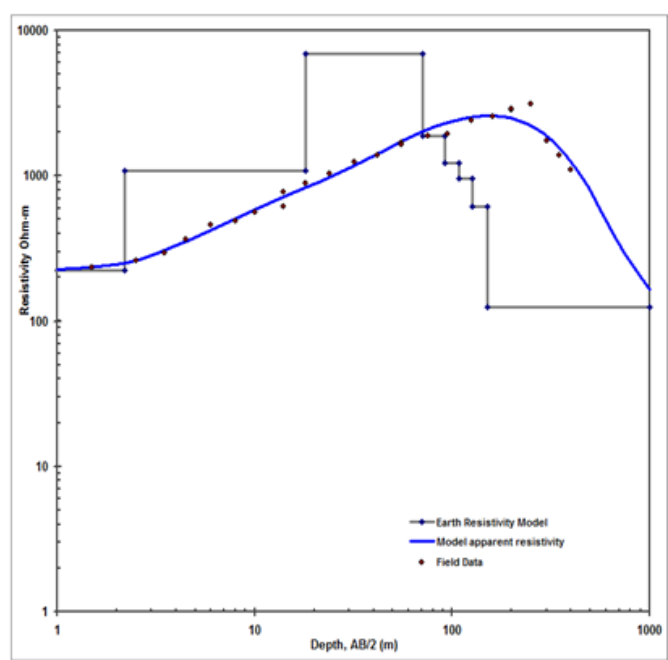

(d)

Figure 4: Typical representative geo-electric curves generated from the resistivity data of the study area: (a)Umulolo, Okigwe (b) Ovim, Isikwuato (c) Ubaha Nneato (4) Anara Mbano 


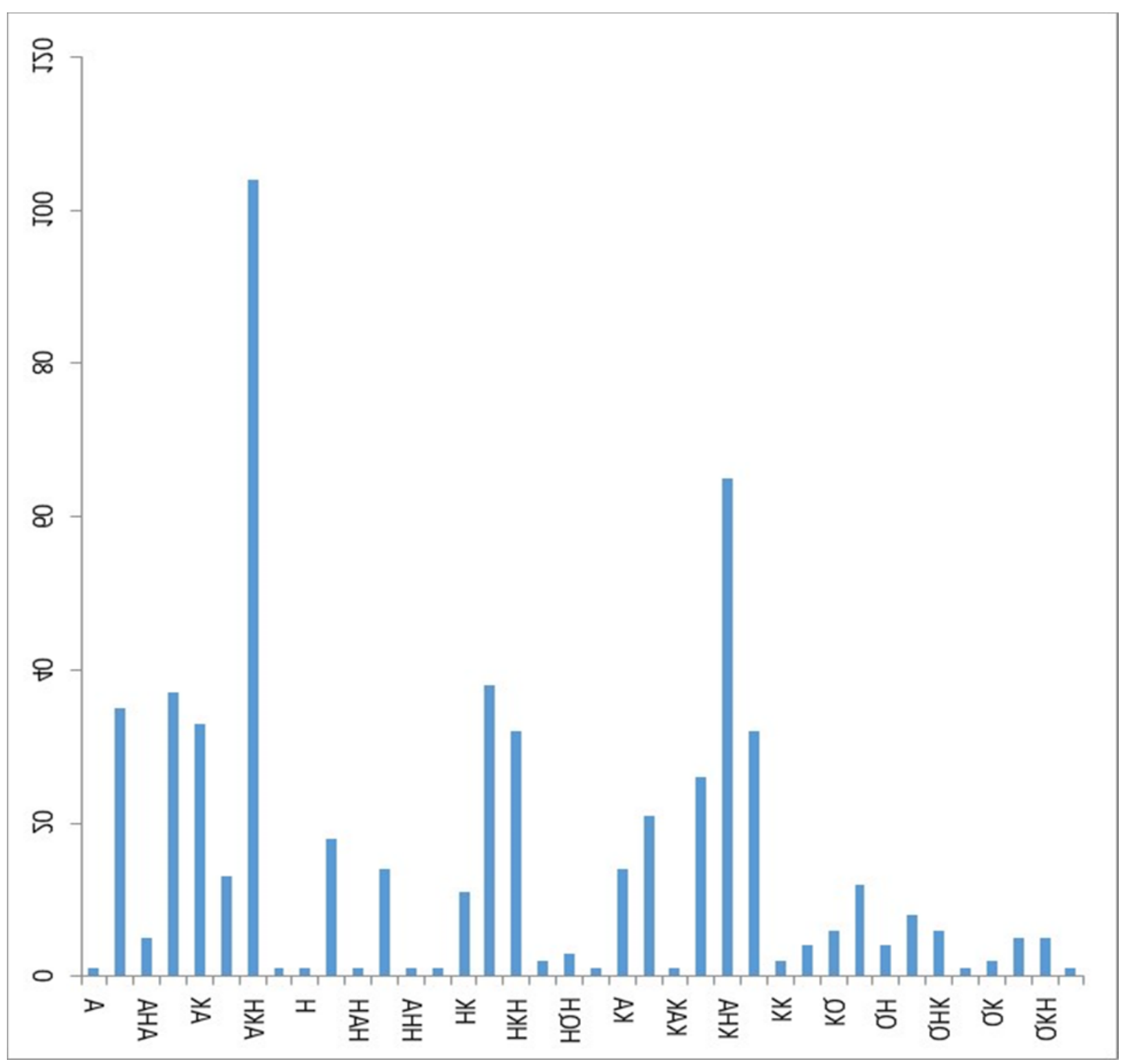

Figure 5: Bar Chart showing various curve types in the study area

Table 2: Statistical representation of curve type in the study area

\begin{tabular}{|c|c|c|c|}
\hline S/N & Curve Type & Frequency & Percentage (\%) \\
\hline & A & 1 & 0.17574692 \\
\hline & AH & 35 & 6.15114236 \\
\hline & AHA & 5 & 0.87873462 \\
\hline & AHK & 37 & 6.5026362 \\
\hline & AK & 33 & 5.79964851 \\
\hline & AKA & 13 & 2.28471002 \\
\hline & AKH & 104 & 18.2776801 \\
\hline & AQ & 1 & 0.17574692 \\
\hline & H & 1 & 0.17574692 \\
\hline & HA & 18 & 3.16344464 \\
\hline & HAH & 1 & 0.17574692 \\
\hline & HAK & 14 & 2.46045694 \\
\hline & HHA & 1 & 0.17574692 \\
\hline & HHQ & 1 & 0.17574692 \\
\hline & HK & 11 & 1.93321617 \\
\hline & HKA & 38 & 6.67838313 \\
\hline & HKH & 32 & 5.62390158 \\
\hline & HQ & 2 & 0.35149385 \\
\hline
\end{tabular}


Citation: Terhemba Theophilus Emberga, et al., Regional Hydro-geophysical Study of the Groundwater potentials of the Imo River Basin Southeastern Nigeria using Surficial Resistivity Data. Australian Journal of Basic and Applied Sciences, 13(8): 76-94.DOI: 10.22587/ajbas.2019.13.8.12

\begin{tabular}{|l|c|c|c|}
\hline & K & 1 & 0.17574692 \\
\hline & KA & 14 & 2.46045694 \\
\hline & KAH & 21 & 3.69068541 \\
\hline & KAK & 1 & 0.17574692 \\
\hline & KH & 26 & 4.56942004 \\
\hline & KHA & 65 & 11.4235501 \\
\hline & KHK & 32 & 5.62390158 \\
\hline & KK & 2 & 0.35149385 \\
\hline & KKH & 4 & 0.7029877 \\
\hline & KQ & 6 & 1.05448155 \\
\hline & KQK & 12 & 2.10896309 \\
\hline & QH & 4 & 0.7029877 \\
\hline & QHA & 8 & 1.4059754 \\
\hline & QHK & 6 & 1.05448155 \\
\hline & QJK & 1 & 0.17574692 \\
\hline & QK & 2 & 0.35149385 \\
\hline & QKA & 5 & 0.87873462 \\
\hline & QKH & 5 & 0.87873462 \\
\hline & QKK & 1 & 0.17574692 \\
\hline
\end{tabular}

The AKH-type curves are the most prevailing in the study area representing about $18.28 \%$ of the total number of curve types. This is followed by the KH-type (11.42\%). The overall signature of the curves represents alternating sequences of resistiveconductive layers. Table 3 is the summary of the layer parameters interpreted from the VES data. Geo-electric layers interpreted ranges from 5 to 10 layers as shown in table 3 .

Table 3. Representative Results of Interpreted Layer Parameters from the Study Area

\begin{tabular}{|c|c|c|c|c|c|c|}
\hline V & $\begin{array}{c}\text { No. } \\
\text { ES }\end{array}$ & \multicolumn{2}{|c|}{ Layer Resistivity $\rho(\Omega \mathrm{m})$} & Layer depth $\mathbf{d}(\mathrm{m})$ & $\begin{array}{c}\text { Layer Thickness h } \\
(\mathrm{m})\end{array}$ & $\begin{array}{c}\text { Cur } \\
\text { ve }\end{array}$ \\
\cline { 1 - 1 } & & &
\end{tabular}

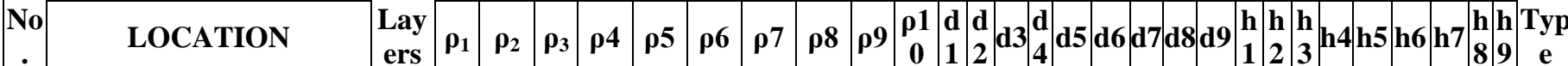

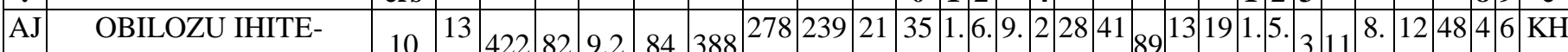

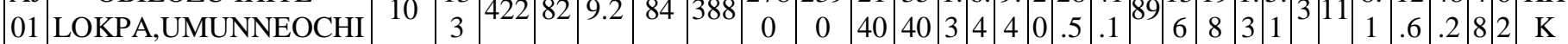

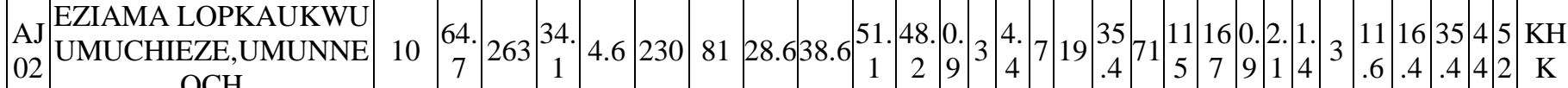

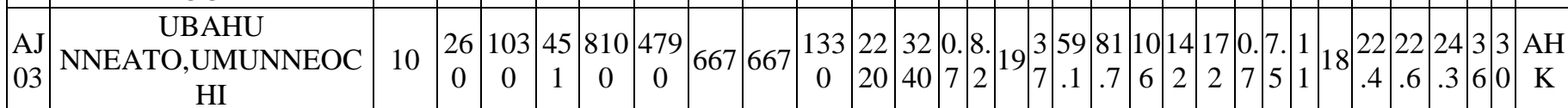

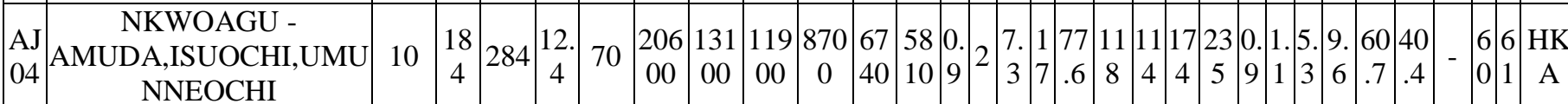

\begin{tabular}{c|c} 
ELUAMA \\
AJ
\end{tabular} LOKPOUKWU

05 UMUCHIEZE,UMUNNE

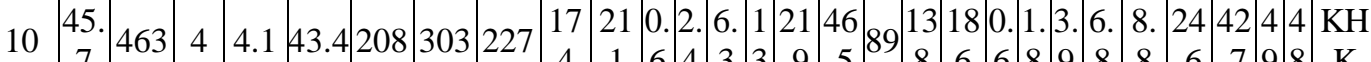
$\mathrm{OCHI}$

\begin{tabular}{|c|c|c|c|c|c|c|c|c|c|c|c|c|c|c|c|c|c|c|c|c|c|c|c|c|c|c|}
\hline $\begin{array}{l}\mathrm{A} \\
\mathrm{M} \\
40\end{array}$ & UMUDIMOHA -AMIKE & 5 & $\begin{array}{c}69 \\
8\end{array}$ & 450 & $\begin{array}{c}68 \\
2\end{array}$ & $\begin{array}{c}698 \\
8\end{array}$ & $\begin{array}{c}134 \\
5\end{array}$ & - & - & - & - & - & \begin{tabular}{c|c}
0. & 3 \\
7
\end{tabular} & $\begin{array}{l}6 . \\
5\end{array}$ & & - & - & - & - & \begin{tabular}{l|l|}
0. & 2. \\
7 & 7
\end{tabular} & \begin{tabular}{cc|}
3. & 13 \\
5 & 9
\end{tabular} \mid & - & - & - & & $\mathrm{AH}$ \\
\hline $\begin{array}{l}\mathrm{A} \\
\mathrm{M} \\
41\end{array}$ & UMUZIKE,UMUOBA 1 & 9 & $\begin{array}{c}88 \\
0\end{array}$ & $\begin{array}{c}262 \\
0\end{array}$ & $\begin{array}{c}\# \# \\
\#\end{array}$ & $\mid \begin{array}{c}162 \\
0\end{array}$ & $\begin{array}{c}245 \\
0\end{array}$ & $\begin{array}{c}411 \\
0\end{array}$ & $\begin{array}{c}659 \\
0\end{array}$ & $\begin{array}{c}369 \\
0\end{array}$ & $\begin{array}{l}13 \\
60\end{array}$ & - & $\begin{array}{l}0.8 \\
4 \\
8\end{array}$ & 17 & $\begin{array}{ll}3 & 6 \\
9 & .\end{array}$ & \begin{tabular}{c|c}
60 & 8 \\
.5 & 8
\end{tabular} & & \begin{tabular}{c|c}
3 & 18 \\
8 & 4
\end{tabular} & - & \begin{tabular}{c|c|}
0. & 8 \\
4 & 4
\end{tabular} & $\mid$\begin{tabular}{l|l}
8. & 22 \\
2 &
\end{tabular} & \begin{tabular}{r|r}
21 & 2 \\
.5 &
\end{tabular} & \begin{tabular}{r|r}
27 & 4 \\
.9 &.
\end{tabular} & $\begin{array}{r}49 \\
.6\end{array}$ & & $\begin{array}{c}\mathrm{AK} \\
\mathrm{H}\end{array}$ \\
\hline $\begin{array}{l}\mathrm{A} \\
\mathrm{M} \\
42\end{array}$ & OGBERURU & 6 & $\begin{array}{l}35 \\
10\end{array}$ & $\begin{array}{c}830 \\
0\end{array}$ & $\begin{array}{l}11 \\
80\end{array}$ & 840 & $\begin{array}{c}356 \\
0\end{array}$ & $\begin{array}{c}800 \\
0\end{array}$ & - & - & - & - & \begin{tabular}{l|l}
1 & 2 \\
1 & 0
\end{tabular} & 34 & $\mid \begin{array}{lll}5 & 8 \\
8 & .\end{array}$ & $\begin{array}{c}83 \\
.5\end{array}$ & - & - & - & \begin{tabular}{ll|}
1 & 8. \\
1 & 7
\end{tabular} & \begin{tabular}{ll|}
1 & 24 \\
&
\end{tabular} & $\begin{array}{l}25 \\
.5\end{array}$ & - & - & - & HA \\
\hline $\begin{array}{l}\mathrm{A} \\
\mathrm{M} \\
43\end{array}$ & ONUNKWO UMUELE & 6 & $\begin{array}{c}59 \\
8\end{array}$ & $\begin{array}{c}736 \\
0\end{array}$ & $\begin{array}{c}59 \\
8\end{array}$ & $\begin{array}{c}306 \\
0\end{array}$ & $\begin{array}{c}140 \\
0\end{array}$ & $\begin{array}{c}107 \\
0\end{array}$ & - & - & - & - & \begin{tabular}{l|l}
8. & 3 \\
7 & 8
\end{tabular} & $\begin{array}{c}10 \\
3\end{array}$ & & & - & - & - & \begin{tabular}{l|l|}
8. & 2 \\
7 & 9
\end{tabular} & $\begin{array}{ll}6 \\
5\end{array} \mid 28$ & 56 & - & - & & $\begin{array}{c}\text { AK } \\
\text { H }\end{array}$ \\
\hline $\begin{array}{l}\text { A } \\
\text { M } \\
44\end{array}$ & $\begin{array}{c}\text { UMUDIM UMUELE } \\
\text { AMAZANO }\end{array}$ & 10 & $\begin{array}{l}38 \\
60\end{array}$ & $\begin{array}{c}233 \\
0\end{array}$ & $\begin{array}{c}40 \\
6\end{array}$ & $\mid \begin{array}{c}302 \\
0\end{array}$ & $\begin{array}{c}121 \\
00\end{array}$ & $\begin{array}{c}118 \\
00\end{array}$ & $\begin{array}{c}170 \\
0\end{array}$ & $\begin{array}{c}920 \\
0\end{array}$ & $\begin{array}{l}64 \\
30\end{array}$ & $\begin{array}{l}50 \\
00\end{array}$ & \begin{tabular}{c|c}
0. \\
5 \\
5
\end{tabular} & 9. & \begin{tabular}{l|l}
2 & 6 \\
7 &.
\end{tabular} & \begin{tabular}{c|c}
66 & 1 \\
.3 &
\end{tabular} & & \begin{tabular}{l|l}
5 & 20 \\
4 & 4
\end{tabular} & $\begin{array}{c}25 \\
6\end{array}$ & \begin{tabular}{c|c|c|}
0. & 2. \\
5 & 4
\end{tabular} & $\begin{array}{lll}7 & 17\end{array}$ & $\begin{array}{r}36 \\
.4\end{array}$ & \begin{tabular}{c|c}
38 & 5 \\
.7 & -5
\end{tabular} & $52 \mid 5$ & \begin{tabular}{ll|l}
5 & 5 \\
0 & 2
\end{tabular} & $\begin{array}{c}\mathrm{AH} \\
\mathrm{K}\end{array}$ \\
\hline
\end{tabular}


Citation: Terhemba Theophilus Emberga, et al., Regional Hydro-geophysical Study of the Groundwater potentials of the Imo River Basin Southeastern Nigeria using Surficial Resistivity Data. Australian Journal of Basic and Applied Sciences, 13(8): 76-94.DOI: 10.22587/ajbas.2019.13.8.12

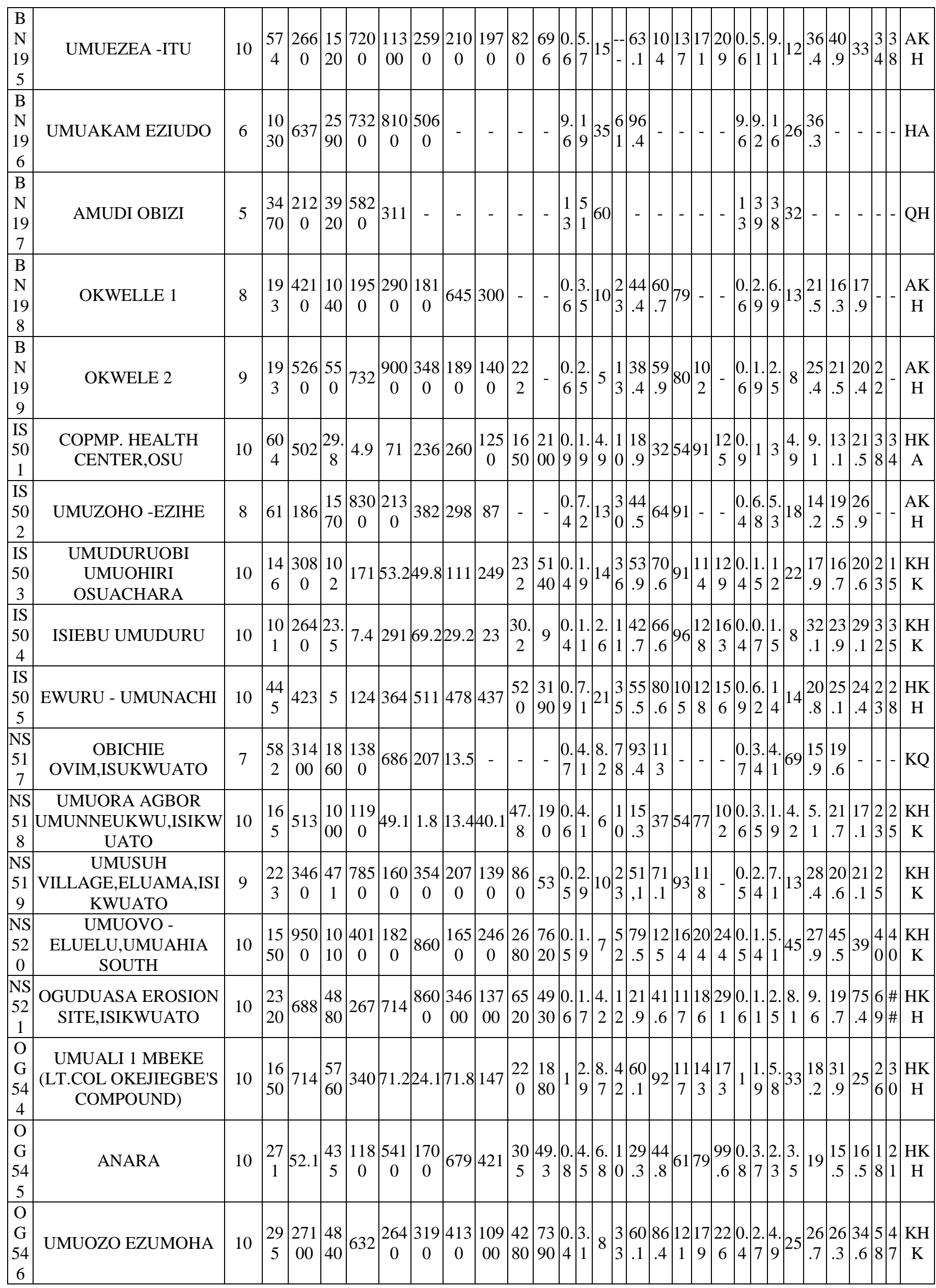




\begin{tabular}{|c|c|c|c|c|c|c|c|c|c|c|c|c|c|c|c|c|c|c|c|c|c|c|c|c|c|c|c|}
\hline $\begin{array}{c}O \\
G \\
54 \\
7\end{array}$ & $\begin{array}{l}\text { UMUEZEALA- } \\
\text { UMUDURU }\end{array}$ & 7 & $\begin{array}{c}74 \\
1\end{array}$ & $\begin{array}{c}166 \\
0\end{array}$ & $\begin{array}{l}26 \\
20\end{array}$ & $\begin{array}{c}322 \\
00\end{array}$ & $\begin{array}{c}720 \\
0\end{array}$ & $\begin{array}{c}304 \\
0\end{array}$ & 733 & - & - & - & \begin{tabular}{l|l}
0. & 3. \\
6 & 2
\end{tabular} & 2.6 & $\mid \begin{array}{l}2 \\
0\end{array}$ & $\begin{array}{r}39 \\
.7\end{array}$ & $\begin{array}{c}74 \\
.3\end{array}$ & - & - & & $\begin{array}{l}0 . \\
6 \\
6\end{array}$ & $\begin{array}{ll}2 . & 14 \\
9 & \end{array}$ & $\begin{array}{r}19 \\
.5\end{array}$ & $\begin{array}{l}34 \\
.6\end{array}$ & - & -- & $\begin{array}{c}\mathrm{AK} \\
\mathrm{H}\end{array}$ \\
\hline $\begin{array}{c}\mathrm{O} \\
\mathrm{G} \\
54 \\
8\end{array}$ & $\begin{array}{c}\text { UMULOLO- } \\
\text { OBOH,OSUAMA }\end{array}$ & 8 & $\begin{array}{c}50 \\
2\end{array}$ & $\begin{array}{c}441 \\
0\end{array}$ & $\begin{array}{l}10 \\
30\end{array}$ & 40.8 & 232 & $\begin{array}{c}172 \\
0\end{array}$ & 170 & 101 & - & - & $\frac{0 .}{5}{ }^{2}$ & 219 & $\mid \begin{array}{l}4 \\
2\end{array}$ & \begin{tabular}{r|r}
62 & 1 \\
.1 &
\end{tabular} & \begin{tabular}{c|c}
15 & 2 \\
2 &
\end{tabular} & $\begin{array}{c}24 \\
3\end{array}$ & - & - & \begin{tabular}{l|l}
0. & 1. \\
5 & 5
\end{tabular} & \begin{tabular}{|l|l|}
1 & 24 \\
7 &
\end{tabular} & $\begin{array}{r}19 \\
.8\end{array}$ & $\begin{array}{r}89 \\
.9\end{array}$ & & -- & $\begin{array}{c}\mathrm{KH} \\
\mathrm{K}\end{array}$ \\
\hline
\end{tabular}

\subsection{Aquifer Resistivity}

Aquifer resistivity across the study area was estimated from the geo-electric curves generated from the Vertical Electrical Sounding data. The aquifer resistivity varied spatially across the study area in line with the geological complexity of the study area. Aquifer resistivity values across the study area varied from as low as $16.38 \Omega$ mto as high as $10,000 \Omega$ mas shown in figure 5 below. These areas with low resistivity are generally believed to be made up of conductive earth materials like clay or shale which may not be good for citing boreholes.

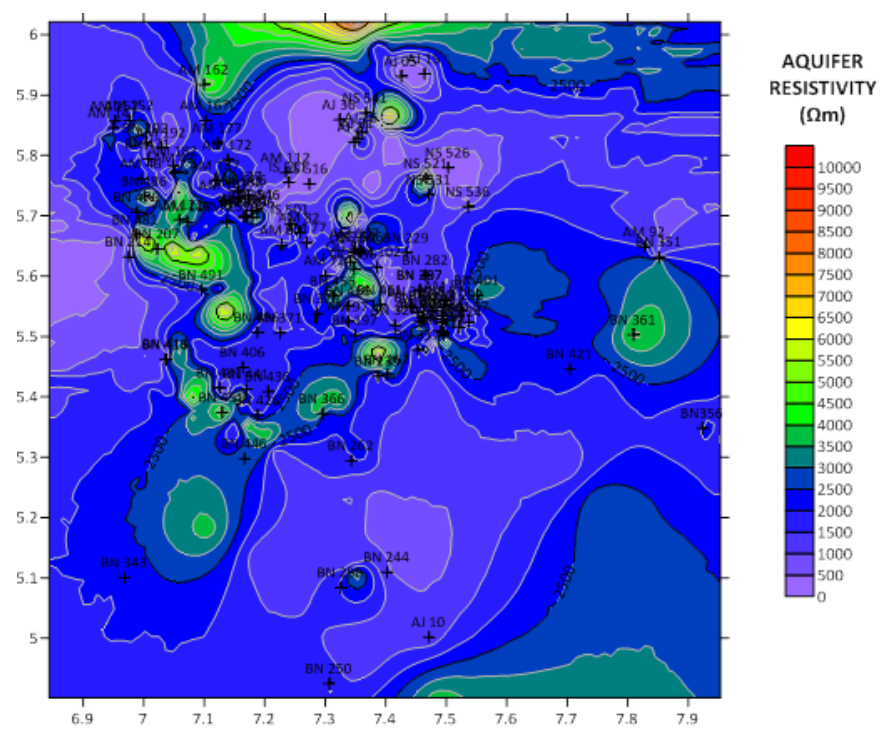

Figure 5:Spatial Variation of Aquifer Resistivity across the study area

\subsection{Aquifer Conductivity}

Aquifer conductivity across the study area was derived by taking the inverse of the aquifer apparent resistivity values. The calculated aquifer conductivity values for all the sounding locations were therefore contoured to generate a spatial map of aquifer conductivity in the study area (Figure 6). The study area revealed a near homogenous conductive region with very low conductivity values with values between $0-0.005 \mathrm{Sm}^{-1}$ to $0.065 \mathrm{Sm}^{-1}$ (Figure 6 ). The areas slightly below the northern part of the study area appears to have fairly high aquifer conductivity with Eluama Lokpoukwu areas having the highest value of $0.0629 \mathrm{Sm}^{-1}$ These low conductivity areas may not be good for groundwater exploration and exploitation.

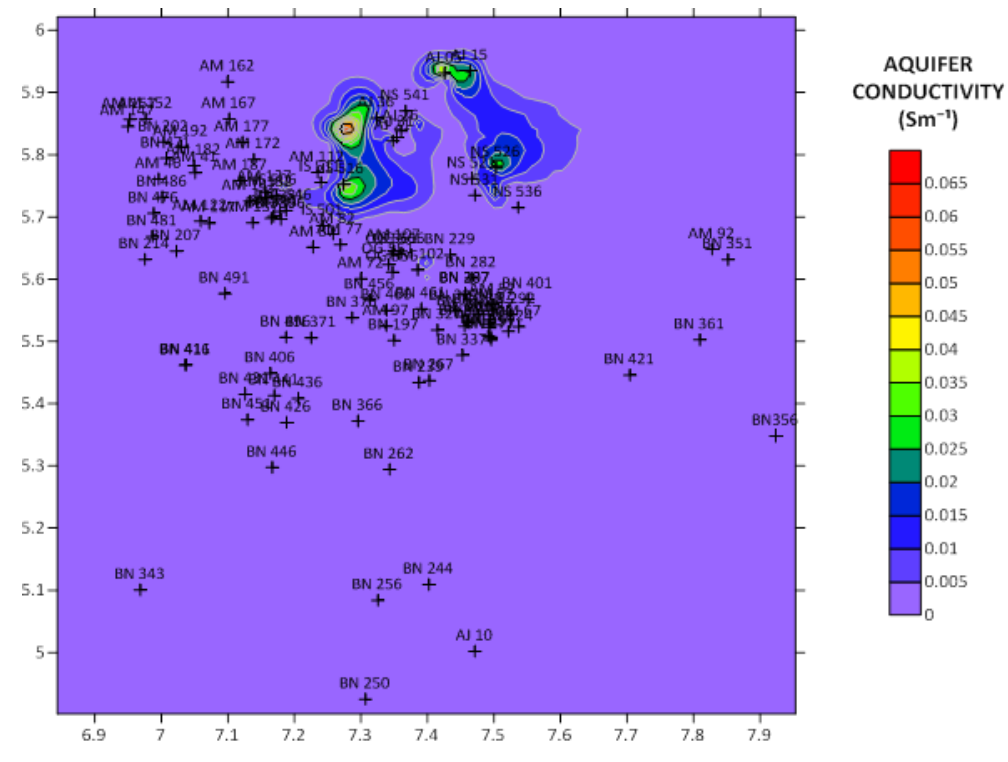


Citation: Terhemba Theophilus Emberga, et al., Regional Hydro-geophysical Study of the Groundwater potentials of the Imo River Basin Southeastern Nigeria using Surficial Resistivity Data. Australian Journal of Basic and Applied Sciences, 13(8): 76-94.DOI: 10.22587/ajbas.2019.13.8.12

Figure 6: Spatial Variation of Aquifer Conductivity across the study area

\subsection{Iso- resistivity (Resistivity Depth Slices) Interpretation across the study area}

Based on the well-established assumption that the effective depth of transmission is approximately equal to $2 / 3$ of half the current electrode separation $(\mathrm{AB} / 2)$, the resistivity depth slices of across the study area were used to generate Iso-resistivity values (Table 4). Several iso-resistivity maps showing depth variations of resistivity at specified intervals of $\mathrm{AB} / 2$ of $5 \mathrm{~m}$, $40 \mathrm{~m}$, $100 \mathrm{~m}, 150 \mathrm{~m}, 250 \mathrm{~m}, 300 \mathrm{~m}, 350 \mathrm{~m}, 400 \mathrm{~m}$, and $500 \mathrm{~m}$ were thus generated as shown in table 4 and figure 7 . The generated maps revealed a high consistency with the western part having very high resistivity with values between 20,000-65,000 $\Omega \mathrm{m}$. There seems to be a fairly progressive decrease in resistivity with depth suggesting a highly resistive overburden and upper layers to less resistive (or conductive) layers at deeper intervals. However, it must be noted that an iso-resistivity map is a qualitative interpretation tool which shows possible variations in resistivity with depth at the given electrode spacing across a region but may not give the true resistivity of a definite or unit geo-electric layer (Mbonu et al., 1991).

Table 4: Representative of selected Downward Continuation of Resistivity across the Study Area

\begin{tabular}{|c|c|c|c|c|c|c|c|c|c|c|c|c|c|c|c|c|}
\hline \multirow{2}{*}{ VES. No. } & \multirow{2}{*}{ Lat. $\left({ }^{0} \mathbf{N}\right)$} & \multirow{2}{*}{ Long. $\left({ }^{0} \mathbf{E}\right)$} & \multicolumn{14}{|c|}{$\mathrm{AB} / 2(\mathrm{~m})$} \\
\hline & & & 5 & 10 & 15 & 40 & 60 & 100 & 150 & 200 & 250 & 300 & 350 & 400 & 450 & 500 \\
\hline AJ 01 & $5^{0} 59.001$ & 727.314 & 210 & 200 & 140 & 40 & 50 & 70 & 100 & 140 & 145 & 170 & 180 & 220 & 260 & 300 \\
\hline AJ 02 & 54.960 & $7^{0} 26.255$ & 130 & 80 & 45 & 50 & 60 & 65 & 62 & 61 & 58 & 56 & 55 & - & - & - \\
\hline $\mathrm{AJ} 03$ & 556.612 & 718.470 & 700 & 800 & 850 & 1000 & 1400 & 1800 & 2000 & 2100 & 2200 & 2300 & 2400 & 2450 & 2500 & 2600 \\
\hline AJ 04 & N5 51.484 & 724.085 & 70 & 30 & 35 & 60 & 90 & 190 & 200 & 300 & 600 & 650 & 700 & 800 & 900 & 1000 \\
\hline AJ 05 & 555.854 & 725.554 & 170 & 100 & 40 & 12 & 18 & 30 & 35 & 40 & 60 & 70 & 80 & 95 & 100 & 110 \\
\hline AM 40 & N5 46.824 & 704.089 & 2200 & 2800 & 3200 & 4000 & 3100 & 3000 & 2900 & 3050 & 3150 & $1 / 0$ & 8150 & 3120 & 3000 & 2950 \\
\hline AM 41 & N5 46.253 & 703.054 & 3500 & & & & & & & & & 100 & 30 & & & 4100 \\
\hline AM 42 & N5 49.993 & 701.385 & 610 & 800 & 1000 & 1900 & 220 & 28 & 5 & 240 & 231 & 2200 & 1300 & 1600 & 1550 & 1400 \\
\hline AM 43 & N5 59.4 & 659.293 & 800 & 700 & 750 & 150 & $0=$ & & $\pi$ & 30 & 5500 & 5000 & 8950 & 0050 & & 5500 \\
\hline & N5 39.564 & E6 59.051 & 1100 & 1300 & & & & & & & & & & & & 400 \\
\hline BN 195 & N5 28 & E7 20.087 & 1300 & 2000 & 250 & 300 & 350 & 50 & 50 & 30 & 3000 & 500 & 2000 & 1000 & 1700 & 1000 \\
\hline & & & & & & & & & & & & & & & & 5500 \\
\hline BN 197 & N5 3 & E7 2 & 3200 & 3200 & 310 & 300 & 280 & 2700 & 2750 & 250 & 2000 & 1800 & 1600 & 1500 & 1400 & 1000 \\
\hline & $N 54$ & & & 1600 & 1700 & 1000 & & & & 1200 & 1100 & 950 & 900 & 500 & 400 & 300 \\
\hline BN 199 & N5 4 & E7 1 & 1200 & 1400 & 1500 & 2000 & 2700 & 2800 & 3000 & 2900 & 2850 & 1700 & 700 & 600 & 550 & 500 \\
\hline & N5 4 & E7 14 & 100 & 9 & 8 & 25 & 40 & 70 & 100 & 105 & 120 & 125 & 200 & 250 & 300 & 350 \\
\hline IS 502 & N5 4 & E7 1 & 190 & 200 & 250 & 700 & 1000 & 1100 & 1100 & 1050 & 1000 & 950 & 700 & 650 & 200 & 140 \\
\hline 503 & N5 4 & E7 13 & 800 & 700 & 400 & 150 & 140 & 130 & 135 & 140 & 200 & 250 & 300 & 350 & 400 & 450 \\
\hline 504 & N5 4 & E7 1 & 400 & 200 & 40 & 25 & 40 & 70 & 80 & 75 & 60 & 55 & 50 & 30 & 25 & 20 \\
\hline IS 505 & N5 4 & E7 1 & 450 & 350 & 180 & 16 & 18 & 28 & 40 & 70 & 80 & 100 & 120 & 140 & 160 & 200 \\
\hline & N5 4 & E7 3 & 2700 & 5000 & 5500 & 4000 & 2000 & 1100 & 900 & 500 & 300 & 200 & 40 & 35 & 30 & 20 \\
\hline NS 518 & N5 46.207 & E7 28.332 & 450 & 510 & 510 & 190 & 11 & 8 & 9 & 12 & 13 & 16 & 17 & 20 & 25 & 30 \\
\hline & N5 54.925 & E7 26.010 & 1200 & 1250 & 1150 & 1600 & 2100 & 300 & 340 & 350 & 3300 & 2900 & 2700 & 1700 & 1600 & 1500 \\
\hline 520 & N5 2 & E7 28. & 4200 & 3000 & 220 & 300 & 320 & 330 & 325 & 250 & 2400 & 3100 & 3300 & 3800 & 4000 & 4500 \\
\hline NS 521 & .693 & E7 28.011 & 1500 & 1800 & 1100 & 800 & 1000 & 2000 & 2500 & 2800 & 3000 & 4000 & 5000 & 5500 & 6000 & 6500 \\
\hline OG 544 & N5 41.441 & E7 13.051 & 1200 & 1500 & 1050 & 1000 & 450 & 160 & 90 & 95 & 100 & 120 & 140 & 160 & 190 & 200 \\
\hline OG 545 & N5 42.929 & E7 09.6093 & 650 & 110 & 150 & 400 & 600 & 800 & 850 & 840 & 830 & 700 & 650 & 400 & 250 & 200 \\
\hline OG 546 & $\mathrm{~N} 542584$ & & 3500 & 4000 & 4500 & 3000 & 1900 & 1600 & 1700 & 2100 & 2200 & 2700 & 2800 & 2900 & 3000 & 4000 \\
\hline OG 547 & N5 41.056 & E7 14.577 & 1500 & 2500 & 3500 & 8000 & 8500 & 7000 & 5500 & 3000 & 1500 & 1300 & 1100 & 1000 & 950 & 900 \\
\hline OG 548 & N5 43.752 & E7 10.649 & 1700 & 1600 & 1100 & 600 & 200 & 150 & 160 & 170 & 180 & 200 & 250 & 300 & 350 & 400 \\
\hline
\end{tabular}

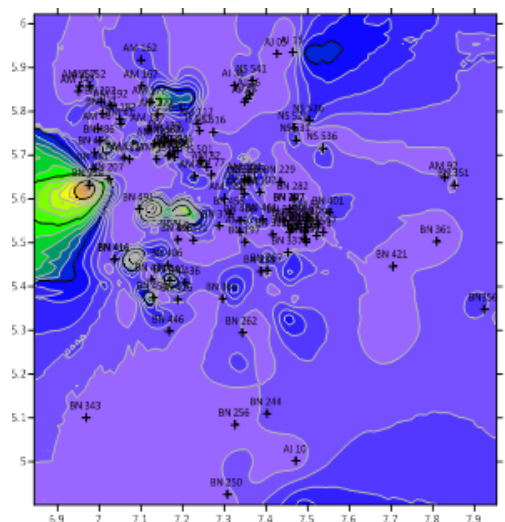

(a)

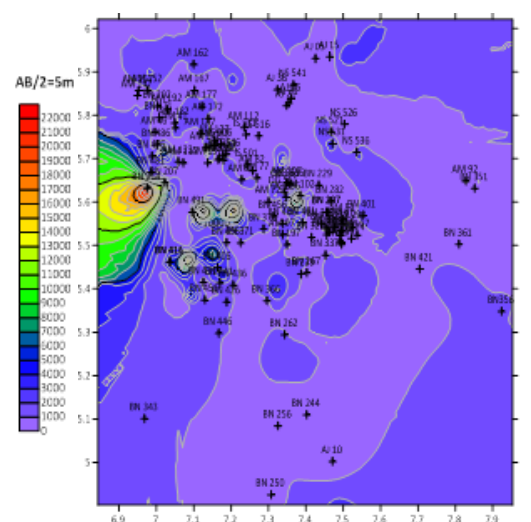

(b)

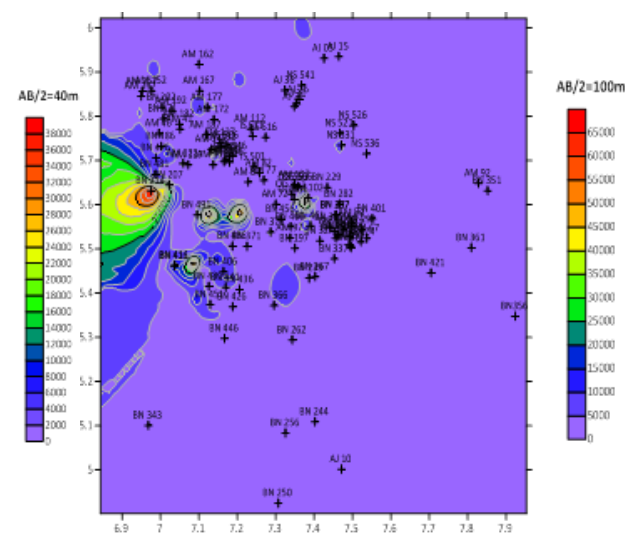

(c) 


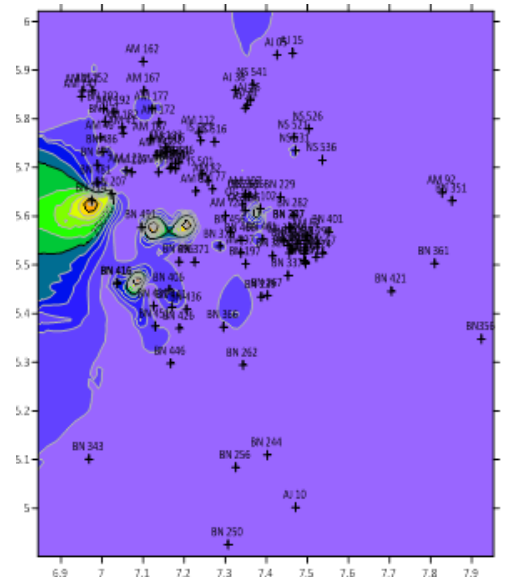

(d)

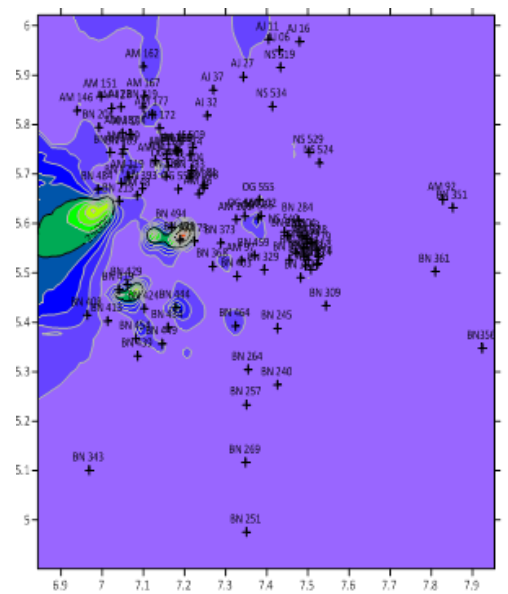

(g)

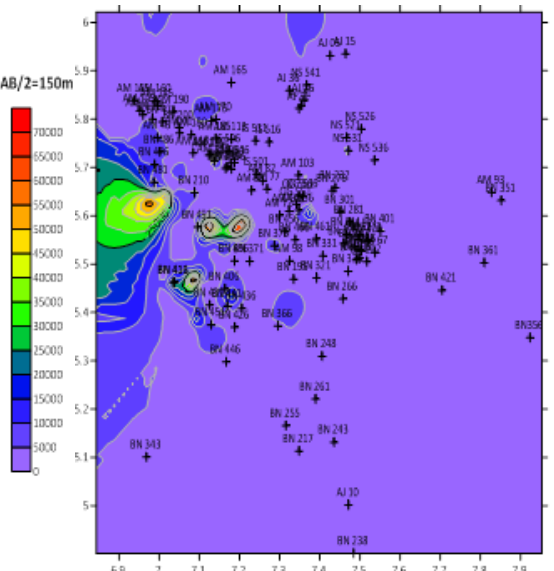

(e)

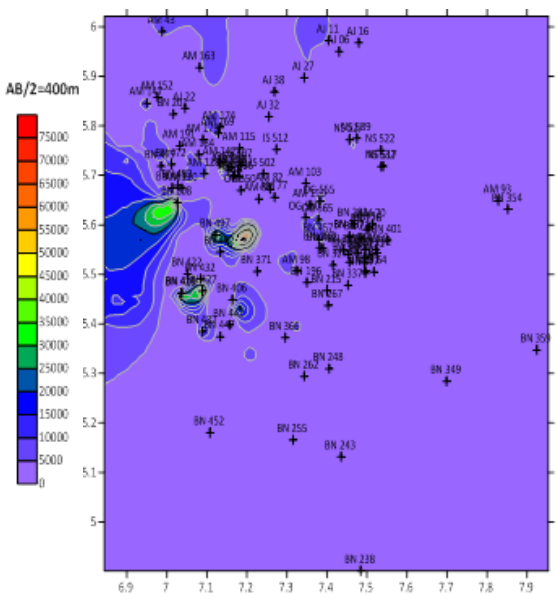

(h)

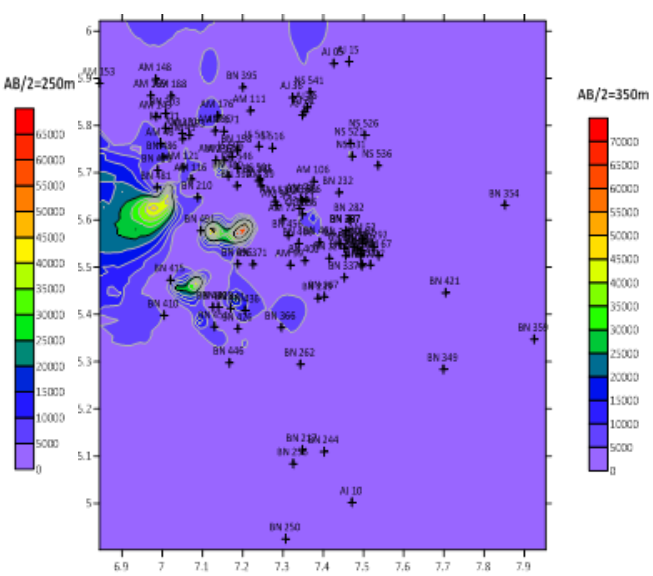

(f)

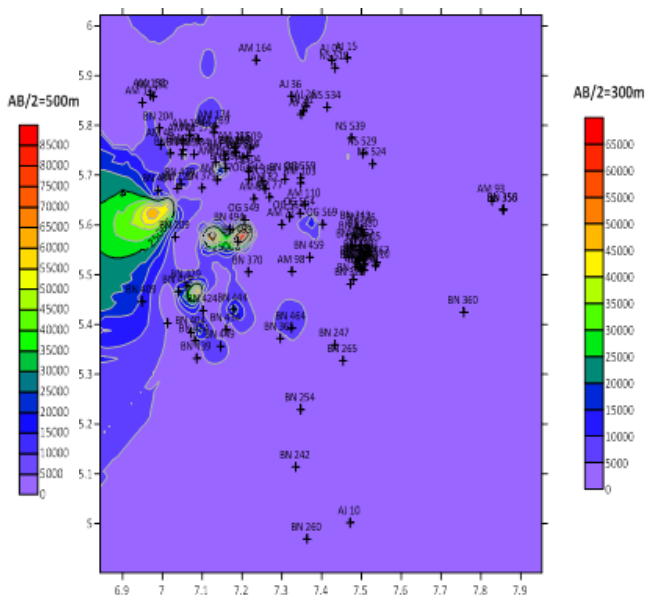

(i)

Figure 7: Iso -resistivity modelling of the study area: (a) $A B / 2=5 \mathrm{~m}$ (b) $A B / 2=40 \mathrm{~m}$ (c) $A B / 2=100 \mathrm{~m}(\mathrm{~d}) \mathrm{AB} / 2=150 \mathrm{~m}$ (e) $\mathrm{AB} / 2$ $=150 \mathrm{~m}(\mathrm{f}) \mathrm{AB} / 2=250 \mathrm{~m}(\mathrm{~g}) \mathrm{AB} / 2=350 \mathrm{~m}(\mathrm{~h}) \mathrm{AB} / 2=400 \mathrm{~m}$ (i) $\mathrm{AB} / 2=500 \mathrm{~m}$

\subsubsection{Iso-resistivity Map $(\mathrm{AB} / 2=5 \mathrm{~m})$}

The generated iso-resistivity map for $\mathrm{AB} / 2=5 \mathrm{~m}$ shown in figure 7arevealed that most parts of the study area especially within the northern, eastern, southwestern and the central areas are underlain by relatively low resistive materials with the exception of the western area. These low resistive areas with magenta color codes in figure 7a have resistivity values ranging between $0-1000 \Omega \mathrm{m}$. The Iso-resistivity model of the study area at this half current electrode spacing with effective depth of penetration of $3.33 \mathrm{~m}(10.87 \mathrm{ft})$ generally revealed that across the study area, the resistivity values increased from about $200 \Omega \mathrm{m}$ (magenta) to $38,000 \Omega \mathrm{m}$ (red). The range of resistivities shown by the magenta color may be an indication of silt/ shale/clay units while the other colors in the map may represent areas underlain by sand/sandstone units.

\subsubsection{Iso-resistivity $\mathrm{Map}(\mathrm{AB} / \mathbf{2}=\mathbf{4 0} \mathrm{m})$}

The iso-resistivity contour map for $\mathrm{AB} / 2=40 \mathrm{~m}$ in figure $7 \mathrm{~b}$ below shows that some parts of the study area within the northern, eastern and southwestern axes are underlain by relatively low resistive materials. The resistivity of the study area at this spacing with sufficient depth of penetration $26.67 \mathrm{~m}(86.93 \mathrm{ft})$ shows that the resistivity of sounding points increases from value > 0 (magenta) to about $56000 \Omega \mathrm{m}$ (red).

Low/medium resistivity values of the range of $0-2000 \Omega m$ (in magenta color) was noticed within the southern, eastern,

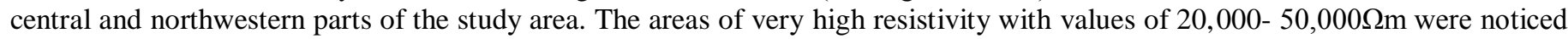
to be restricted to the western part of the map of the study area while the areas represented by blue colours have medium resistivity values ranging between $5000-20,000 \Omega \mathrm{m}$. The range of resistivities shown by the magenta color may be an indication of silt/ shale/clay units while the other colors in the map may represent areas underlain by sand/sandstone units.

\subsubsection{Iso-resistivity Map $(\mathrm{AB} / 2=100 \mathrm{~m})$}

The iso-resistivity map for $\mathrm{AB} / 2=100 \mathrm{~m}$ in figure $7 \mathrm{c}$ below shows that most parts of the study area are underlain by relatively low resistive materials. The resistivity of the study area at this spacing with effective depth of penetration of $66.67 \mathrm{~m}$ revealed that the resistivity values at the sounding points increase from 0 to as high as $65,000 \Omega \mathrm{m}$. At this depth range, the study area became more shaly as over $95 \%$ of the study area is covered by low to medium resistivities (areas covered by magenta

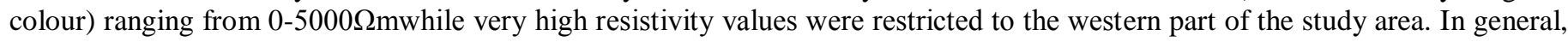


the range of resistivities shown by the magenta color may be an indication of silt/ shale/clay units while the other colors in the map may generally represent areas underlain by sand/sandstone units.

\subsubsection{Iso-resistivity Map $(\mathrm{AB} / 2=150 \mathrm{~m})$}

The iso-resistivity map for $\mathrm{AB} / 2=150 \mathrm{~m}$ in figure 7drevealed that most parts of the study area within the northern, eastern and southwestern axes are underlain by relatively low resistive materials. The resistivity of the study area at this spacing with effective depth of penetration given as $100 \mathrm{~m}(\approx 326 \mathrm{ft})$ revealed that the degree of shaliness increased across the area as the areas covered by magenta colours (representing low-medium resistivities) increased across the study area. The magenta coloured areas have resistivity values ranging between $0-5000 \Omega \mathrm{m}$. High resistivities with values ranging between $5000-50,000 \Omega \mathrm{m}$ are generally restricted to the western axis of the study area.

\subsubsection{Iso-resistivity Map $(\mathrm{AB} / 2=250 \mathrm{~m})$}

The iso-resistivity map for $\mathrm{AB} / 2=250 \mathrm{~m}$ as shown in figure 7erevealeda similar pattern with the values at the depth $\mathrm{AB} / 2=$ $150 \mathrm{~m}$ with most of the area underlain by relatively low-to-medium resistive materials. These low-medium resistivities layers are represented by the magenta colour. The effective depth of penetration at this depth interval is $166.67 \mathrm{~m}$ with the resistivity values varying between $0-55,000 \Omega \mathrm{m}$. Similarly, it was revealed that the degree of shaliness increased with depth across the study area

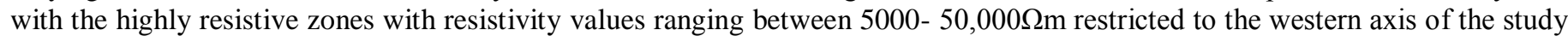
area. These areas with high resistivities possibly represent sand/sandstone units.

\subsubsection{Iso-resistivity $\mathrm{Map}(\mathrm{AB} / 2=300 \mathrm{~m})$}

The iso-resistivity map for $\mathrm{AB} / 2=300 \mathrm{~m}$ is shown in figure $7 \mathrm{f}$. This map revealed a similar pattern with the values at the depth $\mathrm{AB} / 2=250 \mathrm{~m}$ with most of the area underlain by relatively low-to-medium resistive materials. Similarly, it was revealed that amount of shale/clay units increased with depth across the study area with the highly resistive zones with resistivity values

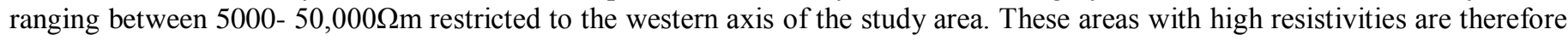
believed to be sand/sandstone units.

\subsubsection{Iso-resistivity $\mathrm{Map}(\mathrm{AB} / 2=350 \mathrm{~m})$}

The iso-resistivity map for $\mathrm{AB} / 2=350 \mathrm{~m}$ in figure $7 \mathrm{~g}$ revealed a similar pattern with the values at the depth $\mathrm{AB} / 2=300 \mathrm{~m}$ with most of the area underlain by relatively low-to-medium resistive materials. Similarly, it was also revealed that the amount of shale/clay units increased at this depth with the high resistive zones restricted to the western axis of the study area. These areas with high resistivities are therefore believed to be sand/sandstone units.

\subsubsection{Iso-resistivity Map $(\mathrm{AB} / 2=400 \mathrm{~m})$}

The iso-resistivity map for $\mathrm{AB} / 2=400 \mathrm{~m}$ in figure $7 \mathrm{~h}$ revealed a similar pattern with $\mathrm{AB} / 2=350 \mathrm{~m}$. The resistivity values of the study area at this half current electrode spacing with effective depth of penetration given as $266.67 \mathrm{~m}$ also showed a similar pattern with the values at the depth of $\mathrm{AB} / 2=350 \mathrm{~m}$ with most of the area underlain by relatively low-to-medium resistive materials. In addition, it was revealed that the amount of shale/clay units increased at this depth with the high resistive zones restricted to the western axis of the study area. These areas with high resistivities are therefore believed to be sand/sandstone units.

\subsection{9 $\mathrm{AB} / 2=500 \mathrm{~m}$}

The iso-resistivity map for $\mathrm{AB} / 2=500 \mathrm{~m}$ in figure $7 \mathrm{i}$ revealed that most parts of the study area are underlain by relatively low resistive materials. The resistivity values of the study area at this half current electrode spacing with effective depth of penetration given as $333.33 \mathrm{~m}$ also showed a similar pattern with the values at the depth of $A B / 2=350 \mathrm{~m}$ with most of the area underlain by relatively low-to-medium resistive materials. In addition, it was revealed that the amount of shale/clay units increased at this depth with the high resistive zones restricted to the western axis of the study area. These areas with high resistivities are therefore believed to be sand/sandstone units.

\subsection{Spatial variation of Aquifer Geometric Properties \\ 3.5.1 Aquifer Depth}

The spatial map of the aquifer depth across the study area as shown in figure 8revealed a high spatial variation. The aquifer depth ranges from $10 \mathrm{~m}$ around the areas underlain by the Benin Formation to as high as 320m in areas underlain by the Ameki, Nsukka, Ogwasi/Asaba and Imo Shale Formations respectively with a mean value of $89.3 \mathrm{~m}$. The areas with high aquifer depths represented by red colours in the map are generally difficult with respect to groundwater exploitation. 
Figure 8:Aquifer depth map of the study area

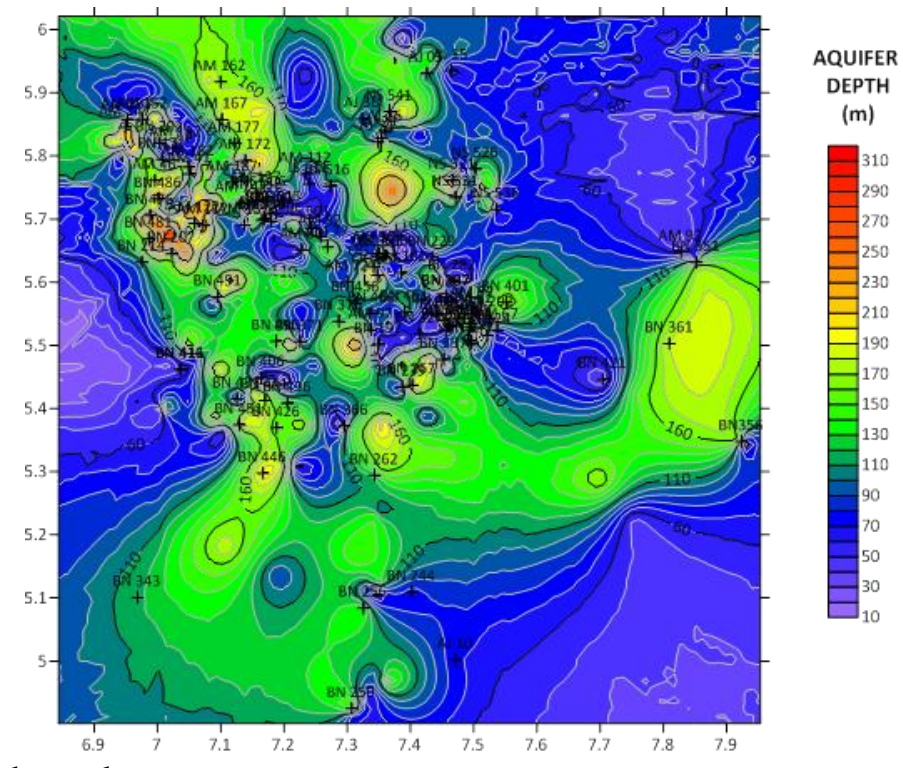

\subsubsection{Aquifer Thickness}

The aquifer thickness across the study area is highly variable as shown in figure 9. Aquifer thickness across the study area ranges from $0-125 \mathrm{~m}$. The spatial variation contour map of the aquifer thickness across the study area thus revealed that the northwestern, part of the northeastern and the northern zones of the study area exhibited high aquifer thicknesses with areas around Aguneze having the highest aquifer thickness with values as high as $130 \mathrm{~m}$ record. Low aquifer thickness values were however recorded in parts of the central, northeastern, southeastern and northern areas of the study area. These low aquifer thickness areas represented with blue colour codes has the lowest value of $2.6 \mathrm{~m}$ around Maranatha Secondary School Umuezealama Mbano. These areas with extremely low aquifer thicknesses might not be very productive and therefore citing of boreholes at the specified depth is not recommended.

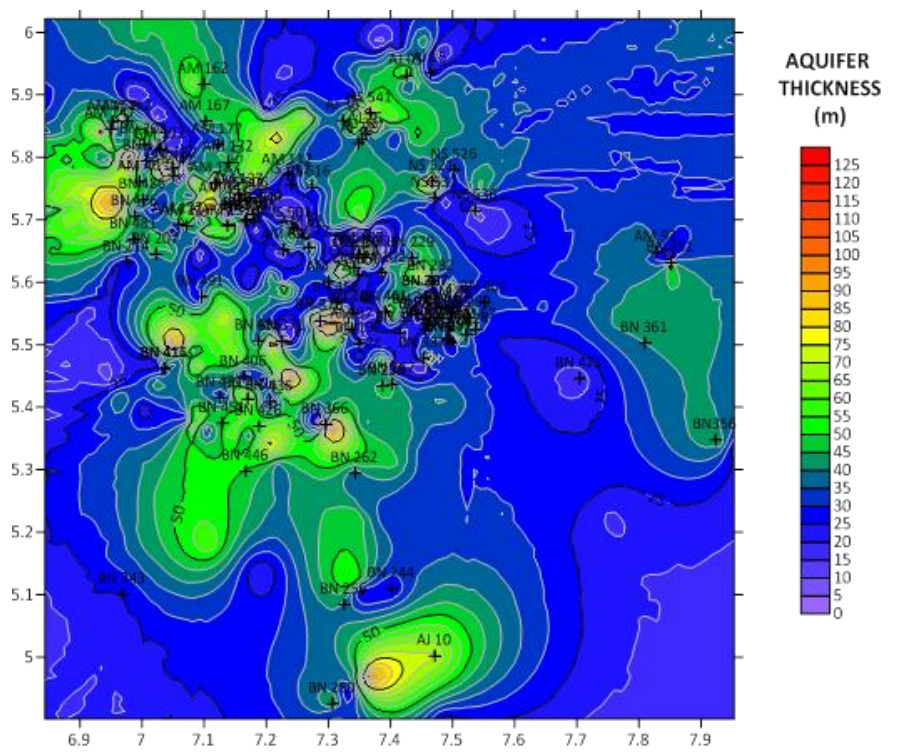

Figure 9: Aquifer thickness map of the study area

\subsubsection{Estimation of Aquifer Lateral Extent}

The geo-electric logs from various locations were correlated with another borehole in formations extracted from litho-logs in drilled boreholes and logs from borehole geophysical logging. The main objective of this correlation is to infer possible spatial variation in geology across the study area (Figure 10). It is believed that this correlation will aid in the understanding of the regional lithostratigraphy of the study area. In addition, to attempt an estimation of the nature, type, lateral extent and continuity of the aquifers of the study area, correlation of the geo-electric sections with litho-logs and electric logs at selected well locations were carried out. A few of these correlations for Nkwofada, Umuechi OgborUgiri Umukabia, Amaraku, and Umuelemai are presented below: 
(a)Nkwofada Arondizogu (AM 165)

A careful analysis of the correlation between the geo-electric section, litho-log and borehole geophysical log at this location revealed a fairly good correlation with respect to the stratigraphy as captured by the various logs (Figure 10a). The topsoil with a resistivity value of $368 \Omega \mathrm{m}$ was interpreted as lateritic ironstone. Hydro-stratigraphically, whereas the geo-electic section delineated the aquiferous layer to be between the depth range of 99-175 m with a thickness $75 \mathrm{~m}$, the borehole litho-log revealed that the aquifer is at the depth range $72-90 \mathrm{~m}$ with a thickness of $18 \mathrm{~m}$. However, the electric log showed that the aquifer lies between the depths of 52-88 m with a thickness of $36 \mathrm{~m}$ (Figure 10a). However, while the results of the borehole logs identified the aquiferous units as whitish/grey sandy clay the geo-electric log gave a resistivity value of $2080 \Omega \mathrm{m}$ for the aquifer unit thus suggesting that the aquifer materials are made up of clean sand/sandstones. The borehole logs and the geo-electric section, therefore, showed a fair correlation. The slight variation in this correlation may be as the result of the problems of equivalence and suppression for which the method is associated with(Zohdy.,1976).

\section{(b)Umuehie Ogbor Ugiri (IS 507)}

The first layer in the geo-electric section is interpreted as lateritic soil with apparent resistivity value of $380 \Omega \mathrm{m}$ (Figure $10 \mathrm{~b}$ ). The interpretation of the correlation assigned a resistivity value of $2,050 \Omega \mathrm{m}$ to the aquiferous layer. It further revealed the aquifer geo-material to of this layer to be composed of sand/sandstone with a thickness of $48 \mathrm{~m}$ (lying between the depth ranges of 76$124 \mathrm{~m})$. On the other hand, the litho-log and the electric-logs revealed that the aquiferous units are made up of a brownish mixture of clay and sand with a thickness of $6 \mathrm{~m}$ (lying between the depth ranges of 75-88 m) and $16 \mathrm{~m}$ (lying between the depth ranges of 88-104m) respectively. Similarly, the geo-electric section, the litho-log and the electric log revealed a fairly good correlation.

(c)Umukabia, Ohuu (BN 226)

At this location which is underlain by the Benin Formation, the geo-electric log identified the aquifer geo-material to be made of sand/sandstones with a thickness $59 \mathrm{~m}$ (lying between the depth ranges of 29-88 m).This layer was assigned a resistivity value of 1,250 $\Omega \mathrm{m}$. Similarly, the borehole logs identified the aquiferous units as whitish sand of thickness $12 \mathrm{~m}$ (lying between the depth ranges of $26-38 \mathrm{~m}$ ) for the strata-log and $18 \mathrm{~m}$ (lying between the depth ranges of 20-38 $\mathrm{m}$ ) for the electric-log. The borehole $\operatorname{logs}($ litho-log and electric $\log$ ) and the geo-electric log displayed a good correlation (Figure 10c). In addition, the both the borehole $\operatorname{logs}$ and the geo-electric log revealed that the identified aquiferous unit may be a confined aquifer bounded by shale/clay layers.

\section{(d)Amaraku, IsialaMbano (AM 80)}

The first layer of the geo-electric section with resistivity $212 \Omega \mathrm{m}$ is interpreted as reddish lateritic sand (figure 10d). The aquiferous layer as identified by the geo-electric section is sand with a thickness of $45 \mathrm{~m}$ (at the depth range of 62-107 $\mathrm{m}$ ) and resistivity value of $1360 \Omega \mathrm{m}$. The strata-log identified the aquifer unit as made up of fine grained whitish sand of thickness $30 \mathrm{~m}$ (lying between the depths of 78-108 m). The geo-electric log therefore correlated perfectly well with that of the strata-log. The aquifer units as indicated by both logs are underlain by an electrically conductive layer of shale. Amaraku and its environments, by this correlative interpretation therefore is believed to be prolific in groundwater potentials.

\section{(e)Umuelemai, Mbano(BN 391)}

The geo-electric log as this location identified the aquifer unit as composed of sand/sandstone with a thickness of $28 \mathrm{~m}$ (between the depth ranges of 78-106 $\mathrm{m}$ ). The resistivity value of this layer is given as $1760 \Omega \mathrm{m}$. Similarly, the strata-log also identified the aquifer geo-material as sandstone with a thickness of $16 \mathrm{~m}$ (at the depths of 30-46 $\mathrm{m}$ ). There is therefore a near perfect correlation between the geo-electric section and the strata-log at this location. The aquifer unit as revealed by the geoelectric log is underlain by an electrically conductive layer of shale. 


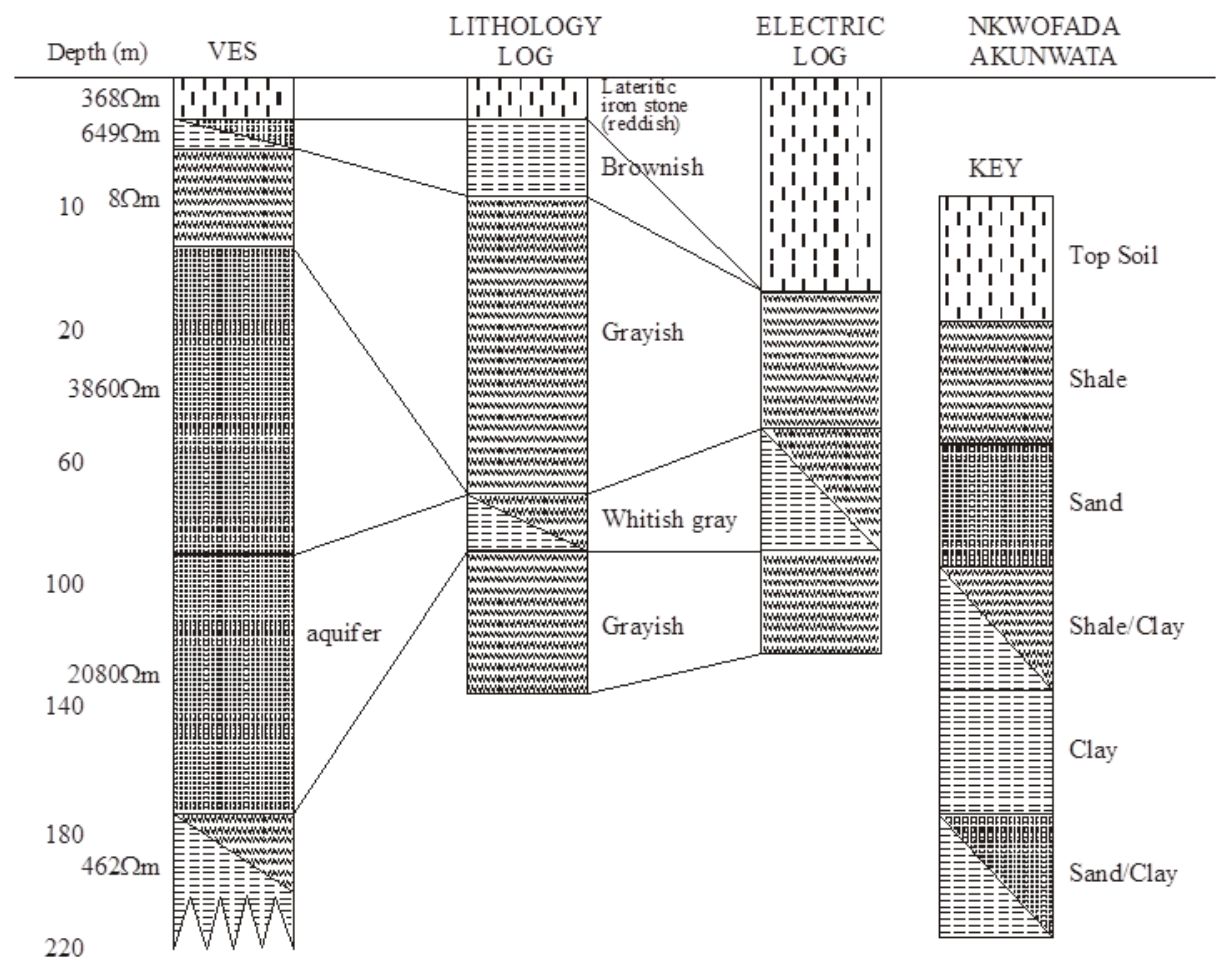

(a)

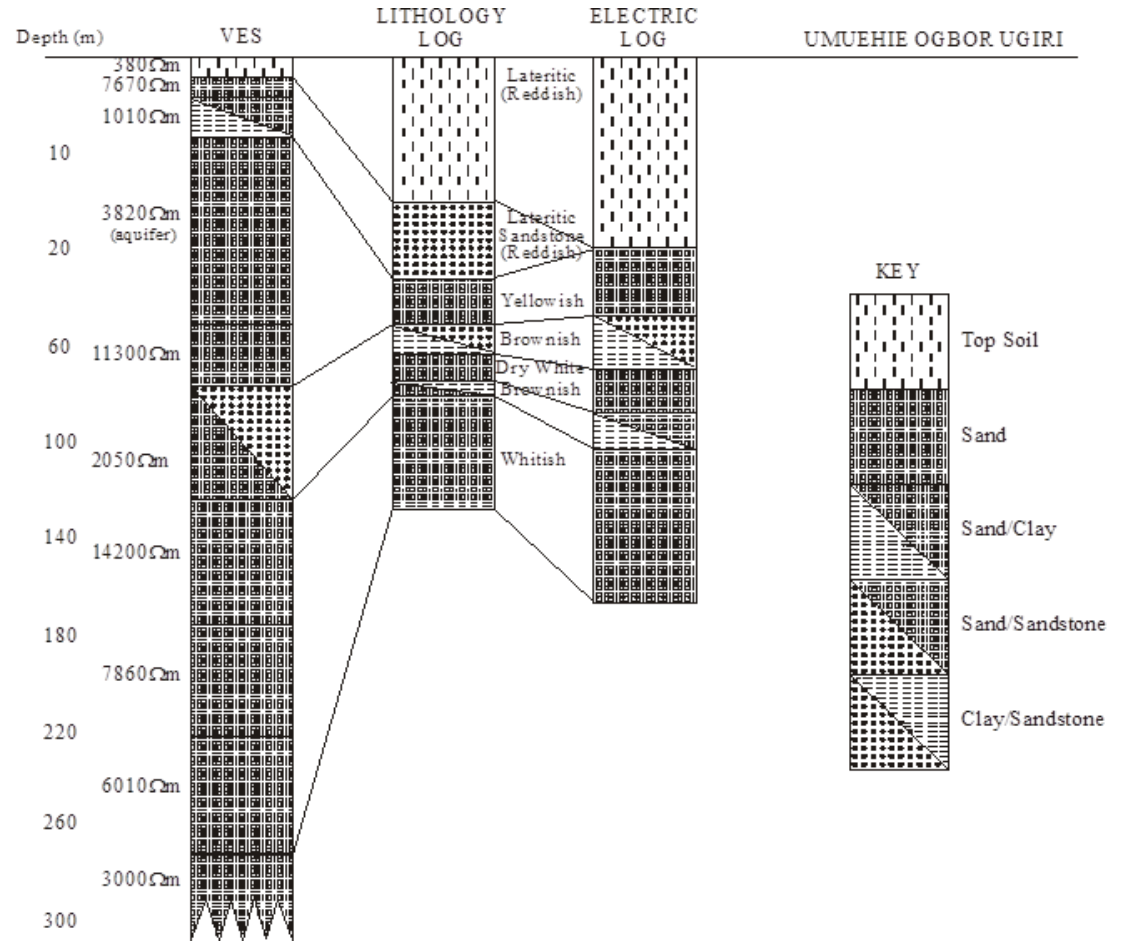

(b) 


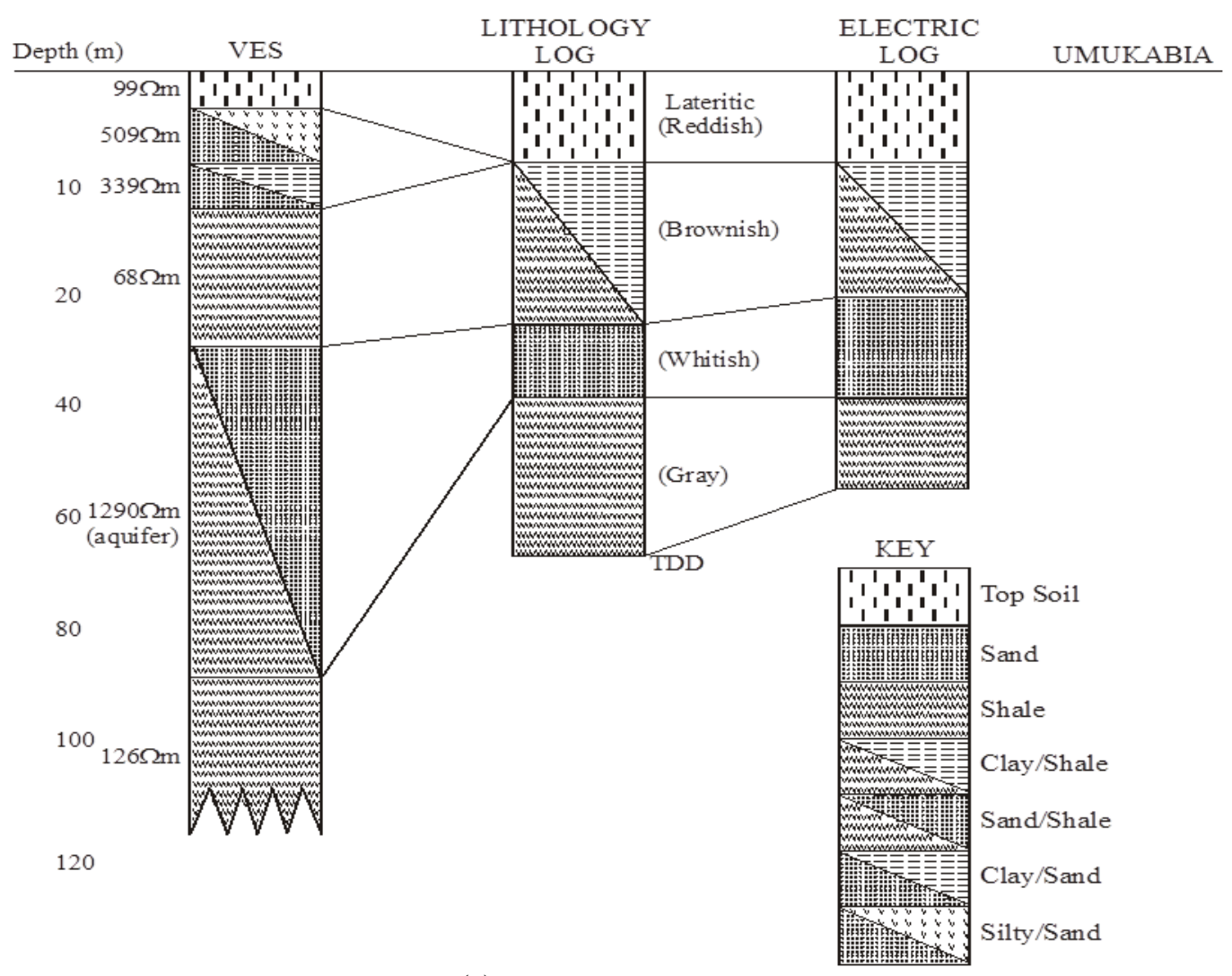

(c)

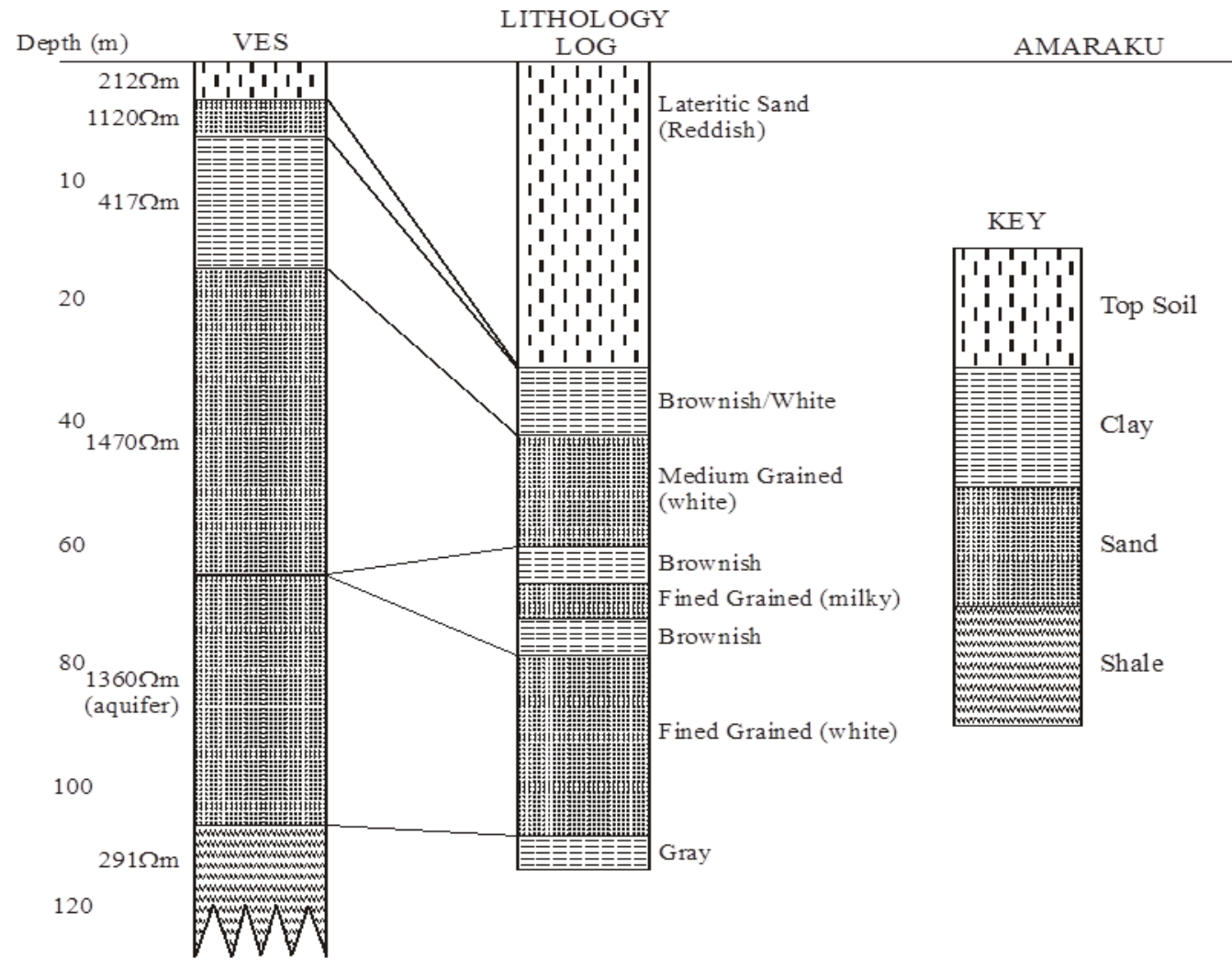

140

(d)

Figure10 (a - d): Correlation of geo-electric log, litho-Log Section and electric-Log of the study area: (a)Nkwofada Akunwata (b) Umuwhie Ogbo Ugiri (c) Umukabia (d)Amaraku (e)Umuelemai Mbano 


\subsection{Dar-Zarrouk Parameters of the study area}

\subsubsection{Transverse Resistance}

Transverse resistance and longitudinal conductance are generally referred to as Dar-zarrouk Parameters. They have found a lot of application in hydrogeophysical and environmental geophysical studies. Transverse resistance is usually estimated in direct current resistivity studies by using the product of aquifer resistivity $(\Omega \mathrm{m})$ and aquifer thickness $(\mathrm{m})$. The transverse resistance contour map of the study area is presented in figure 10 with values ranging from 0 to $75,000 \Omega \mathrm{m}^{2}$. The eastern and northern parts of the study area appear to have low transverse resistances with Okigwe and Onuimo areas having values of $6,006 \Omega \mathrm{m}^{2}$ and 30000 $\Omega \mathrm{m}^{2}$ respectively. These low transverse resistance values correspond to areas in magenta colours on figure 11. Similarly, parts of the central and northwestern areas appear to have high transverse resistance with Nwoagwu Amuda -Isuochi having the highest transverse resistance value of about $225,866.4 \Omega \mathrm{m}^{2}$ which corresponds to the yellow/pink colour coded regions. The areas with magenta colours are likely to be unproductive since their transverse resistance values are low.

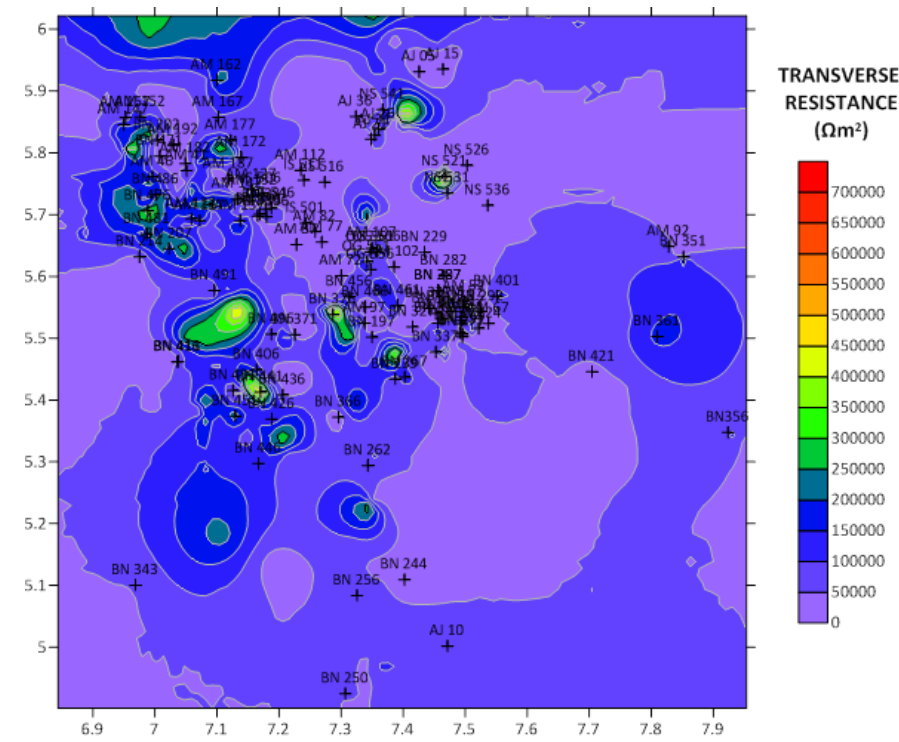

Figure 11: Transverse Resistance map of the study area

\subsubsection{Longitudinal Conductance}

Longitudinal conductance across the study area was estimated by taking the ratio of aquifer thickness (m) to aquifer apparent resistivity $(\Omega \mathrm{m})$. The distribution of longitudinal conductance values across the study area in figure 12 shows that part of the northern and central portions of the study area appear to have high longitudinal conductance especially around Onuimo and Ehime Mbano areas. The southern axes of the study area appear to have low longitudinal conductance with Umuokwara - Ihebinowerre having the lowest value of $0.00096 \Omega^{-1}$. This corresponds to the magenta coloured region. These areas with low longitudinal conductivity may are believed to be very productive. With the exception of these magenta coloured areas, other parts of the study area are expected to be underlain by thick and conductive materials having relatively higher longitudinal conductance thus making the area very poor aquifer materials.

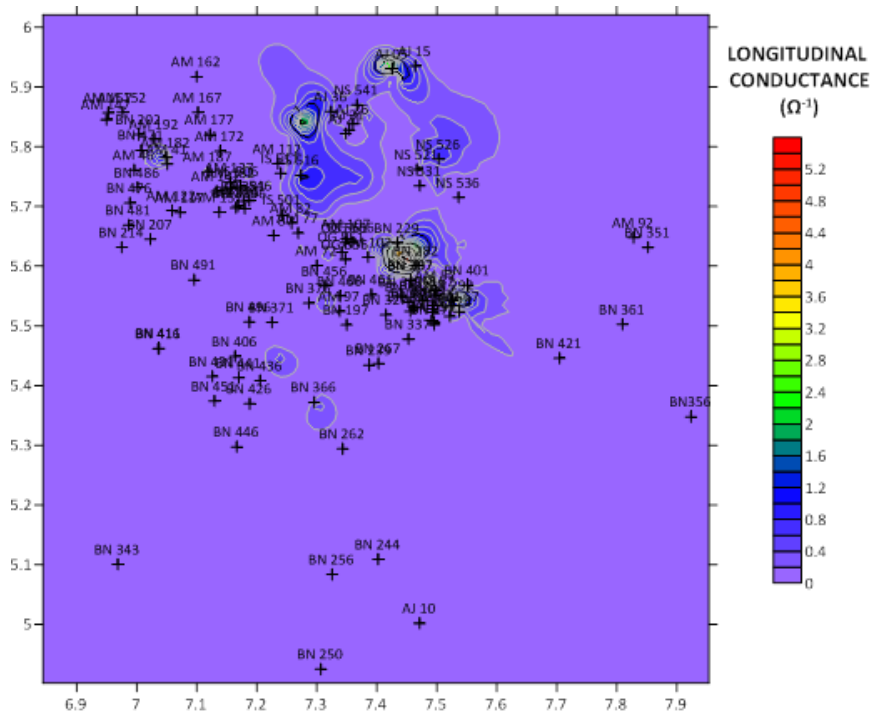

Figure 12:Longitudinal Conductance map of study area 
Citation: Terhemba Theophilus Emberga, et al., Regional Hydro-geophysical Study of the Groundwater potentials of the Imo River Basin Southeastern Nigeria using Surficial Resistivity Data. Australian Journal of Basic and Applied Sciences, 13(8): 76-94.DOI: 10.22587/ajbas.2019.13.8.12

\subsection{SUMMARY, CONCLUSION AND RECOMMENDATION}

The hydro-geophysical study of the Imo River Basin, Southeastern Nigeria using surficial resistivity data have been carried out with the objective of appraising the groundwater potentials of the area. Five hundred and sixty- nine (569) vertical electrical sounding (VES) were carried out with the objective of delineating the aquifer units and appraising their potentials using the $\mathrm{ABEM}^{\mathrm{TM}}$ Terrameter (SAS) 4000.

Results of the study revealed that the geo-electric curves interpreted from the study area include the $\mathrm{A}, \mathrm{AH}, \mathrm{AHA}, \mathrm{AK}, \mathrm{AKH}$, HKA,KHA etc with the dominant curve type being the AKH type. The layer resistivity varies across the study area thus reflecting the geological complexity of the study area with the aquifer resistivity values across the study area varying from as low as $16.38 \Omega \mathrm{m}$ to as high as $10,000 \Omega \mathrm{m}$.Iso-resistivity maps generated at the depth intervals of $\mathrm{AB} / 2 \mathrm{of} 5 \mathrm{~m}, 40 \mathrm{~m}, 100 \mathrm{~m}, 150 \mathrm{~m}, 250 \mathrm{~m}$, $300 \mathrm{~m}, 350 \mathrm{~m}, 400 \mathrm{~m}$, and $500 \mathrm{~m}$ revealed a high consistency with the western part having very high resistivity with values

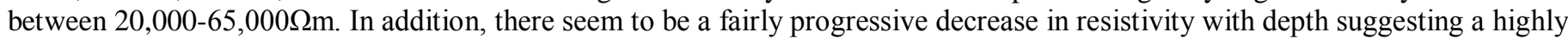
resistive overburden and upper layers to less resistive (or conductive) layers at deeper intervals. Aquifer depth ranges from 10m around the areas underlain by the Benin Formation to as high as $320 \mathrm{~m}$ in the areas underlain by the Ameki, Nsukka, Ogwasi/Asaba and Imo Shale Formations with a mean value of $89.3 \mathrm{~m}$. Aquifer thickness across the study area is variable with a aquifer thickness across the study area ranging from 0 - $125 \mathrm{~m}$. Transverse resistance and longitudinal conductance generally referred to as Dar-zarrouk Parameters were also estimated across the study area. The transverse resistance ranges from 0 to 75,000 $\Omega \mathrm{m}^{2}$ while the distribution of longitudinal conductance values across the study area revealed that he southern axes of the study area appear to have low longitudinal conductance with Umuokwara - Ihebinowerre having the lowest value of $0.00096 \Omega^{-1}$.

The high spatial variation of resistivity across the study area is possibly due to variations in the physic-chemical properties of the formations. These properties include the particle size of materials, porosity/permeability, water content, compaction, and chemical composition of the water as the result of the presence of dissolved solids. Similarly, the close correlation of the interpretation of resistivity data with borehole information is an indication of the applicability of the resistivity data in characterizing aquifers geo-materials. This is very true despite the limitations of the vertical electrical resistivity sounding method especially with respect to the challenges of equivalence and suppression.

The findings of this study revealed that the shale/clay across the entire area increased with depth. In addition, the aquifer potentials of the study area was revealed to be variable with the aquifer type, nature and characteristics generally controlled by the underlying geology. The areas with the highest transverse resistance values are expected to give the highest groundwater yield. Areas for future groundwater development have been suggested on the ground of high transverse resistance values. The Isoresistivity maps revealed that the western section of the study area is the most productive area for ground water exploration. This is because the transverse unit resistance which is a product of aquifer thickness $(\mathrm{h})$ and resistivity $(\Omega)$ are high in this area. It was there concluded that the southern part of the study area has a high aquifer potential when compared to the northern part. In addition, the Benin Formation was delineated as the formation with the greatest aquifer potentials in the study area. It is therefore recommended that a detailed groundwater exploration should be carried out before siting a borehole in the study area.

\subsection{ACKNOWLEDGEMENTS}

The authors are grateful to Anambra Imo River Basin Authority (AIRBA) and GEOVINDA Services, 83 Oyima Street off Anokwuru Street, Owerri Imo State, Nigeria for providing the data used for this research work.

\subsection{REFERENCES}

Aller, L., Bennet, T., Lehr, J.H., Petty, R.J., \&Hacket, G.(1985). DRASTIC: A Standardized System for Evaluating Ground Water Pollution Using Hydrological Settings. Ada, Ok, USA: Prepared by the National Water Well Association for the US EPA office of Research and Development

Al-Rumaih, F. \& Ali, H.O. (1986).Resistivity Measurements for Groundwater Investigation in the Umm Al-Aish Area, Northern Kuwait. Hydrology 88, 185198.

Atkinson, S.F., and Thomlinson, J.R.(1994). An evaluation of ground water pollution potential through GIS modeling. ASPRS/ACSM

Bachmat, Y. \& Collin, M. (1987).Mapping to Assess Groundwater Vulnerability to Pollution. In; W. van Duijvenbooden and H. G. Heigold PC, Gilkeson RH, Cartwright K, Reed PC (1979) Aquifer transmissivity from surficial electrical methods. Gr Water 17(4),338-345

Boerner, F.D., Schopper, J.R.\&Willer, A. (1196). Evaluation of transport and storage properties in the soil and groundwater zone from induced polarization measurements. Geophysics 44, 583-601

Breusse J.J.,(1963). Modern Geophysical Method for Subsurface Water Exploration. Geophysics 28, 633

Dodds, A.R. \&Ivic, D (1988). "Integrated Geophysical Methods Used for Groundwater Studies in the Murray Basin, South Australia". In: Geotechnical and Environmental Studies, SEG Tulsa, OK. 11,303-310

Emberga T.T. (2019): Comparative Analysis of Techniques for Estimating Geo-hydraulic Properties and Aquifer Vulnerability in Imo River Basin, Southeastern Nigeria. Unpublished Ph.D dissertation, Federal University of Technology Owerri, Nigeria

Ejiogu B. C., Opara A. I,. Nwosu E. I ,Nwofor O. K. Onyema J. C.. Chinaka J. C .(2019) Estimates of aquifer geo-hydraulic and vulnerability characteristics of the Imo State and environs, Southeastern Nigeria, using electrical conductivity data. Environ Monit Assess 
Citation: Terhemba Theophilus Emberga, et al., Regional Hydro-geophysical Study of the Groundwater potentials of the Imo River Basin Southeastern Nigeria using Surficial Resistivity Data. Australian Journal of Basic and Applied Sciences, 13(8): 76-94.DOI: 10.22587/ajbas.2019.13.8.12

Ekwe, A. C., Onu, N.N., \&Onuoha, K.M. (2006). Estimation of Aquifer Hydraulic Characteristics from Electric Sounding Data: The case study of Middle Imo River Basin Aquifers, South Eastern Nigeria. Journal of Spatial Hydrology; 6,( 2), $121-132$

Ekwe A.C. and Opara A. I.(2012): Aquifer Transmissivity from Surface Geo-electrical Data: A Case Study of Owerri and Environs, Southeastern Nigeria.JOURNAL GEOLOGICAL SOCIETY OF INDIA Vol.80, July 2012, pp.123-12

Etu-Efeotor, J.O. and Akpokodje E.G.(1990). Aquifer Systems of the Niger Delta. Journal of Mining and Geology 26(2), 279-284

Huntley, D. (1986). Relations between permeability and Electrical Resistivity in granular aquifers. Ground water 24 (4), $466-474$

Ibe, K.M., \&Uzokwu S.C. (2004).An appraisal of subsurface Geology and Groundwater Resources of Owerri and Environs based on electrical resistivity survey and borehole data evaluation.Journal of Environmental monitoring and assessment. 303-321.

Igbokwe, M.U., Okwueze, E. E. \&Okereke, C.S. (2006). Delineation of Aquifer zones from Geoelectric Sounding in Kwa Ibo River Watershed, South Eastern Journal of Engineering and Applied science 1(4),410-421

Koefoed,O. (1977).Geosounding Principles 1. Resistivity Sounding Measurements.ElseriaAmsterdanpp 277.

Kogbe, C.A (1976) ,Paleogeographic History of Nigeria from Albian times. In Kogbe C.A (Ed) ,Geology of Nigeria. Elizabeth Publisher, Lagos 237-282.

Lobo-Ferreira, J.P. \& Oliveira M.M. (1997).DRASTIC groundwater Vulnerability mapping of Portugal, Groundwater: An endangered resource. In proceedings of the 27th congress of the International Association for Hydraulic Research, San Francisco U .S.A, Theme C 132-137.

Lowrie, W. (1997). Fundamentals of Geophysics. Cambridge University Press, New York.

Maillet R (1947) The fundamental equations of electrical prospecting. Geophysics 12,529- 556

Mbonu, P.D.C., Ebeniro, J.O., Ofoegbu, C.O. \&Ekine, A.S. (1991).GeoelectricSounding for the Determination of Aquifer Characteristics in part of the Umuahia Area of Nigeria. Geophysics. 56 ( 2), 284-291.

Okoro, E I; Egboka, B C E; Onwuemesi, A G (2010): Evaluation of the aquifer characteristic of Nanka Sands using hydrogeological method in combination with Vertical Electrical Sounding (VES) Journal of Applied Sciences and Environmental Management World Bank assisted National Agricultural Research Project (NARP) - University of Port Harcourt ISSN: 1119-8362 Vol. 14, Num. 2, 2010, pp. 5-9

Omosuyi, G.O., Ojo, J.S.\&Olorunfemi, M.O. (2008). Geoelectric Sounding to Delineate Shallow Aquifers in the Coastal Plain Sands of Okitipupa Area, South Western Nigeria. The Pacific Journal of Science and Technology, 562-577

Onuoha K.M. \&Mbazi F.C.C., 1988.Aquifer Transmissivity from Electrical Sounding Data. The Case of Ajali Sandstone Aquifers, South-West of Enugu. In: Ofoegbu, C.O(Ed), Ground Water and Mineral Resources of Nigeria, Fried-vieweg and Sohn ,Wiesbaden, 17-29

Onuoha, K.M. \&Mbazi, F.C.C. (1988).Aquifer Transmissivity from Electrical Sounding Data of the Case of Ajali Sandstone Aquifers, South East of Enugu, Nig.. In: C.O. Ofoegbu (ed.). Groundwater and Mineral Resources of Nig. Friedvieweg\& Son Pub.: Berlin, Germany. 17-29.

Onwuegbuche, A.A. (1993). Geoelectrical Investigations in the Imo River Basin, Nigeria. Unpublished Ph.D thesis. Department of Physics, University of Calabar. 2-62.

Opara, A. I. Onu ,N .N. \&Okereafor , D.U. ,2012.Geophysical Sounding for the Determination of Aquifer Hydraulic Characteristic from Dar-Zarrock parameters. Case Study of Ngor-Okpala, Imo River Basin Southeastern Nigeria. The Pacific Journal of Science and Technology 13(1), 590-603.

Rahman A, (2008) A GIS based DRASTIC model for assessing Ground Water Vulnerability in Shallow aquifer in Aligarh India.Applied Geography, 28, 32-53

Sandberg, S.K. (1993). Examples of Resolutions Improvement in Geoelectrical soundings Applied to Groundwater Investigations. Geophysical Prospecting 41, 207-227.

Schwarz S.D (1988). Application of Geophysical Methods to Ground water Exploration in the Tolt River Basin, Washington State: In Geotechnical, Environmental and Engineering. Geophysical Society and European Section, E.E.G.S. 9-12.

Sekhar, M., Mohan-Kumar,M. S.\& Sridharan, K., (1992).Parameter Estimation in an Aquifer Water Table AquitardSystem.Journals of Hydrology. 136, 177 - 192

Telford, W.M., Geldart, L.P., Sheriff, R.E. \& Keys, D.A. (1976) Applied Geophysics. Cambridge University Press, London.

Todd, D.K. (1980). Groundwater Hydrology: John Wiley and Sons Inc., New York.

Ugada, U.,Ibe, K.K.,Akaolisa, C.Z. \&Opara, A.I. (2013). Hydrogeophysical evaluation of aquifer hydraulic characteristics using surface geophysical data: a case study of Umuahia and environs, Southeastern Nigeria

Ukandu, J.S., Udom G.J. \&Nwankwoala, H.O. (2011) Aspects of the Hydrogeology of Umuahia-South Local Government Area, Abia State, Nigeria: Journal of Environmental Research and Management 2(2) 14-26

Uma, K.O. (1989). An appraisal of the Groundwater Resources of the Imo River Basin: Nigerian Journal of Mining and Geology, 25 ( $1 \& 2), 305-315$

Uma, K.O. \&Egboka B.C.E. (1985) Groundwater potentials of Owerri and its environs: Nigerian Journal of Mining and Geology 22, 57-64

US EPA. (1985). DRASTIC: A standard system for evaluating groundwater potential using hydrogeology settings. Ada, Oklahoma WA/EPA series p 163

Vingoe, P. (1972). Electrical Resistivity Surveying ABEM Geophysical Memorandum 5/72, 1-3

Zohdy, A., \& Bisdorf, R.(1989). Programs for the Automatic Process in and aInterpretation of Schlumberger Sounding Curves in QuickBASIC 4.0: U.S. Geological Survey, Open-File Report, 89-137.

Zohdy, A.A.R. (1976). Application of Surface Geophysics (Electrical Methods of Groundwater Investigation): in Techniques for Water Resources investigations in the United States. Section D, Book 2, 5-55 